\title{
A HYBRID SCHEME TO COMPUTE CONTACT DISCONTINUITIES IN ONE-DIMENSIONAL EULER SYSTEMS*
}

\author{
Thierry Gallouët ${ }^{1}$, Jean-Marc Hérard ${ }^{1,2}$ And Nicolas Seguin ${ }^{1,2}$
}

\begin{abstract}
The present paper is devoted to the computation of single phase or two phase flows using the single-fluid approach. Governing equations rely on Euler equations which may be supplemented by conservation laws for mass species. Emphasis is given on numerical modelling with help of Godunov scheme or an approximate form of Godunov scheme called VFRoe-ncv based on velocity and pressure variables. Three distinct classes of closure laws to express the internal energy in terms of pressure, density and additional variables are exhibited. It is shown first that a standard conservative formulation of above mentioned schemes enables to predict "perfectly" unsteady contact discontinuities on coarse meshes, when the equation of state (EOS) belongs to the first class. On the basis of previous work issuing from literature, an almost conservative though modified version of the scheme is proposed to deal with EOS in the second or third class. Numerical evidence shows that the accuracy of approximations of discontinuous solutions of standard Riemann problems is strengthened on coarse meshes, but that convergence towards the right shock solution may be lost in some cases involving complex EOS in the third class. Hence, a blend scheme is eventually proposed to benefit from both properties ("perfect" representation of contact discontinuities on coarse meshes, and correct convergence on finer meshes). Computational results based on an approximate Godunov scheme are provided and discussed.
\end{abstract}

Mathematics Subject Classification. 65M99, 76M15, 76N15, 80A10.

Received: May 2, 2002. Revised: July 31, 2002.

\section{INTRODUCTION}

Computation of gas-liquid flows is of great importance in several industrial fields. For instance, when focusing on nuclear safety problems, two great problems arise. The first one is known as the LOCA (Loss Of Coolant Accident) problem. It corresponds to the unsteady flow of highly pressurised water entering an open domain initially occupied by still air at atmospheric pressure. The resulting flow contains a mixture of water and air, and the thermodynamical behaviour of the medium is quite uneasy to describe and therefore to compute. Another problem corresponds to the ebullition crisis, due to sudden heating of coolant in reactor. The flow suddenly becomes highly unsteady and contains two phases (liquid water for instance and saturated vapour).

\footnotetext{
Keywords and phrases. Godunov scheme, Euler system, contact discontinuities, thermodynamics, conservative schemes.

* The third author has been supported by Electricité de France (EDF) grant under contract C02770/AEE2704. Computational facilities were provided by EDF-Division Recherche et Développement.

${ }^{1}$ LATP-UMR CNRS 6632, C.M.I., Université de Provence, 13453 Marseille Cedex 13, France.

e-mail: gallouet@cmi.univ-mrs.fr, herard@cmi.univ-mrs.fr

2 Département MFTT, Électricité de France - R\&D, 78401 Chatou Cedex, France. e-mail: herard@chi80bk.der.edf.fr, seguin@chi80bk.der.edf.fr
} 
The dynamics of the whole is not very well understood up to now, both from a dynamical point of view and thermodynamical aspect.

Simple models may be proposed in order to try to account for the physics involved in these problems. The most well known is the Homogeneous Equilibrium Model. It only requires to give a suitable equation of state (EOS). This one may be very simple or much more complex and tabulated [38]. It nonetheless requires schemes to compute Euler equations which enable computing strong rarefaction waves, shocks and contact discontinuities. Many schemes have been proposed to deal with that kind of system with reasonable success $[6,13,14,16,47]$, which rely on "standard" upwinding techniques such as those developed to cope with aerodynamics $[22,23,36,39,46]$. Another physically relevant approach relies on the Homogeneous Relaxation Model, which in addition requires computing an extra mass balance equation including (stiff) source terms in order to account for mass transfer terms between phases (see for instance the work of Bilicki and co-workers [7-9]). More complex models may also be suggested to predict two phase flow patterns on the basis of the two fluid approach for instance [27], using the single pressure or the two pressure approach $[21,41,47]$. These a fortiori require better understanding of physical process involved but also urge the development of stable and highly accurate algorithms, due to the occurrence of many different time scales, and to other specific problems including presence of first order non conservative terms and of stiff source terms, conditional hyperbolicity when retaining the single pressure approach. From a numerical point of view, a common need for this kind of problem is to obtain an accurate prediction of contact discontinuities.

We restrict here our attention to the frame of the single fluid approach and Euler type systems in one dimension, and consider the governing set of equations:

$$
\left\{\begin{array}{l}
\frac{\partial W}{\partial t}+\frac{\partial F(W)}{\partial x}=0, \\
W(0, x)=W_{0}(x),
\end{array}\right.
$$

where both $W$ and $F(W)$ take values in $\mathbb{R}^{5}$. The conservative variable $W$ and convective flux $F(W)$ read:

$$
\begin{aligned}
W^{t} & =(\rho, \rho C, \rho U, E, \rho \psi), \\
F(W)^{t} & =\left(\rho U, \rho C U, \rho U^{2}+P, U(E+P), \rho \psi U\right) .
\end{aligned}
$$

The total energy $E$ is written in terms of the kinetic energy plus the internal energy $\rho e$ :

$$
E=\frac{\rho U^{2}}{2}+\rho e
$$

where $e$ is a function of $(P, \rho, C, \psi)$ which is the EOS. We have noted as usual the density $\rho$, the velocity $U$, the pressure $P$, the concentration $C$ and a colour function $\psi . Q=\rho U$ is the momentum. Roughly speaking, $C$ is commonly used in single phase models, and $\psi$ is used in two-phase models. The governing equation for the colour function is more commonly written in non conservative form $\partial_{t} \psi+U \partial_{x} \psi=0$. We nonetheless will privilege the conservative form in order to remove any ambiguity concerning formulation of jump conditions. This equation on colour function is useful in some cases, for instance when modeling stiffened gas EOS. We also denote the specific entropy $s$ as a function of $P, \rho, C, \psi$.

It is now well known that great difficulties in computations of $(1,2)$ arise when attempting at computing shock tube test cases with high pressure ratio and distinct phases on each side of the initial membrane. Part of the difficulty is connected with the need to compute the contact discontinuity with sufficient accuracy. This has already been pointed out in the literature by different workers including Karni [29,30], Abgrall [1] for instance. It clearly appears in preliminary computations that fully conservative schemes such as Godunov scheme [23] provide rather poor accuracy around contact discontinuities, when the EOS is not the basic single component perfect gas EOS, when examinating coarse meshes. This is a particularly annoying point when one aims at providing an a posteriori computation of a discrete gradient of the ratio $T=P / \rho$, which of course requires 


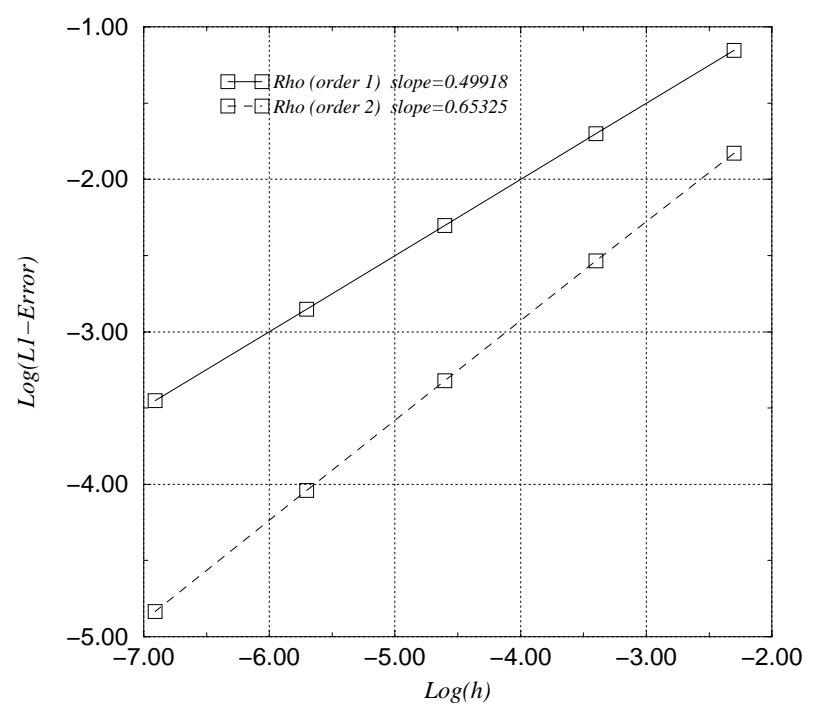

FIGURE 1. L1 norm of the error. Moving contact discontinuity in Euler system (perfect gas EOS).

sufficient accuracy close to the contact discontinuity. Another point which urges for a global effort in this direction is connected with the very small rate of convergence of variables governed by pure advection, say:

$$
\frac{\partial g}{\partial t}+U \frac{\partial g}{\partial x}=0
$$

the measure of which is provided for instance in [18], and is approximately $\frac{1}{2}$ for so called first-order schemes, and $\frac{2}{3}$ for so called second-order schemes, when the initial data is discontinuous. Figure 1 provides a measure of the error in $L^{1}$ norm when computing a pure contact discontinuity of the Euler system of gas dynamics with perfect gas state law.

Actually, several ways to tackle with the problem of moving contact discontinuities have been suggested by Karni [29,30], Abgrall [1], Karni and Abgrall [31], Abgrall and Karni [2], Fedkiw et al. [17], Sethian [43], Saurel and Abgrall [42], and other workers Shyue [44], Allaire, Clerc and Kokh [3,4,33], Lagoutière [34], Barberon, Helluy and Rouy [5,40], ...

We note anyway that focus has actually been given on specific EOS such as mixture of perfect gases, or equivalently on stiffened gas EOS. More recently Van der Waals EOS has been investigated by Shyue [44]. In the latter case, the difference between the model, namely the set of PDE with adequate initial and boundary conditions, and the number of discrete equations which is computed, is not totally clear. More precisely, the exact amount of redundent discrete information, and the specificities due to particular choice of EOS, or of basic flux schemes in the fully conservative schemes, do not clearly arise. In the approach proposed below, it will be seen for instance that the choice of stiffened gas EOS is quite different from the choice of Van der Waals EOS.

The purpose of the present paper is thus the following. It is intended to provide some generic way to compute accurately Euler type systems on coarse meshes and on fine meshes with help of Godunov scheme at least, and if possible with cheaper algorithms in order to cope with the broadest frame of equations of state. Since no theoretical result on convergence is reachable, it seems also of great interest to:

1. provide numerical evidence that the basic Godunov scheme and a sufficiently broad class of approximate Godunov schemes converge for any EOS towards the right solution;

2. examine whether modified "Godunov" schemes converge towards the right solution. 
The presently proposed strategy enables to deal with any EOS in one-dimensional framework. From a practical point of view, one only needs to decompose the EOS in order to distinguish contributions pertaining to three distinct classes. The first class of EOS includes the perfect gas EOS for single component flows and some other very simple laws. The second class contains EOS such as the mixture of perfect gases and the stiffened gas EOS. The third one contains the remaining.

As usual, we define $W_{i}^{n}$ the mean value of the conservative variable $W$ over cell $i$ at time $t^{n}$. We also use the standard definitions: $U_{i}^{n}=Q_{i}^{n} / \rho_{i}^{n}, e_{i}^{n}=\left(E_{i}^{n}-\frac{1}{2} \rho_{i}^{n} U_{i}^{n} U_{i}^{n}\right) / \rho_{i}^{n}, C_{i}^{n}=(\rho C)_{i}^{n} / \rho_{i}^{n}$ and either $\psi_{i}^{n}$ when the colour function is computed with a non conservative equation, or its counterpart $\psi_{i}^{n}=(\rho \psi)_{i}^{n} / \rho_{i}^{n}$ in the conservative case. Throughout the paper we call $p_{i}^{n}$ the pressure on cell $i$ at time $t^{n}$ which is used to compute local one dimensional Riemann problem at each interface. The classical value of pressure used by standard conservative schemes is $p_{i}^{n}=P_{i}^{n}$, where

$$
P_{i}^{n}=P\left(\rho_{i}^{n}, e_{i}^{n}, C_{i}^{n}, \psi_{i}^{n}\right),
$$

with $P(\rho, e, C, \psi)$ the pressure given by the EOS (thus given by an analytic or tabulated law).

Actually, the first class of EOS is perfectly accounted for by standard conservative schemes, when defining discrete pressure $p_{i}^{n}$ as the analytical value of pressure $P_{i}^{n}$. For other EOS, $p_{i}^{n}$ will not be set to $P_{i}^{n}$ in hybrid schemes. The new hybrid algorithm will only require computing one (or two) extra equation(s) (indeed redundent discrete information), depending on the specific form of the EOS. If an extra equation needs to be computed, it is only used to express the discrete value of the pressure $p_{i}^{n}$ at the end of any time step in terms of conservative variables, and additional redundent information.

The outline of the paper is the following. We briefly recall the main properties of the governing set of equations $(1,2)$ of the single-phase or two-phase model assuming equal velocities within each phase in Section 1. In Section 2, closure laws to express internal energy in terms of pressure, density and (possibly) complementary variables including concentrations of species will be detailed, and three distinct classes of EOS will be exhibited. In Sections 3, 4 and 5, we recall the properties of the conservative exact Godunov scheme to deal with conservation laws, or in an alternative way of an approximate conservative Godunov scheme called VFRoe-ncv which is based on velocity and pressure variables $[11,18,20]$, that is to say:

$$
Y^{t}=(\Phi(W))^{t}=(U, P, g(\rho, s), C, \psi),
$$

which depend on the kind of EOS(a short presentation of VFRoe-ncv schemes is provided in the Appendix A). In Section 6, a modified version of the previous fully conservative schemes is proposed in order to improve accuracy of computations on coarse meshes. It will be shown that the whole approach exactly extends those previously proposed by Abgrall, Karni, Saurel and Shyue. Results obtained when computing a single component perfect gas state law, a mixture of perfect gases, Van der Waals EOS are discussed in Section 7. The latter three belong to the three distinct classes. Other computations including EOS with Chemkin database, and any tabulated EOS will be discussed, which again will confirm that accurate approximations of solutions of shock tube experiments may be obtained with any kind of EOS, even when these have some non negligible contribution in the third class. In Section 8, we finally present a blend scheme which benefits from advantages of standard conservative schemes (convergence towards the right solution as the mesh size tends to 0 ) and their hybrid version (accuracy on coarse meshes).

We emphasize that though somewhat similar, the present approach should not be confused with the efficient energy relaxation method proposed by Coquel and Perthame (see [15] and also [25,26]). We also insist that an important ingredient in the method proposed below is that the interface Riemann solver perfectly preserves unsteady contact discontinuities. 


\section{Some Standard RESults For Euler Equations}

We briefly recall here some notations and properties of system (1)

$$
\left\{\begin{array}{l}
\frac{\partial W}{\partial t}+\frac{\partial F(W)}{\partial x}=0 \\
W(0, x)=W_{0}(x)
\end{array}\right.
$$

with both $W$ and $F(W)$ given by $(2,3)$.

The whole system must be complemented with a physically relevant entropy inequality:

$$
\frac{\partial \eta}{\partial t}+\frac{\partial F_{\eta}}{\partial x} \leq 0
$$

The specific entropy $s$ complies with

$$
\hat{\gamma} P \frac{\partial s(P, \rho, C, \psi)}{\partial P}+\rho \frac{\partial s(P, \rho, C, \psi)}{\partial \rho}=0
$$

and allows to define an entropy-entropy flux pair $\left(\eta, F_{\eta}\right)=(-\rho \log s,-\rho U \log s)$.

We introduce the speed of sound waves $c$ following: $\rho c^{2}=(P / \rho-\rho \partial e / \partial \rho)(\partial e / \partial P)^{-1}$ (here we consider $e$ as a function of $P, \rho, C, \psi)$. We assume that $\hat{\gamma} P:=\rho c^{2}$ is positive. Thus the system $(1,2)$ is hyperbolic. It has real eigenvalues and associated right eigenvectors span the whole space $\mathbb{R}^{5}$. Eigenvalues are:

$$
\lambda_{1}=U-c, \lambda_{2}=\lambda_{3}=\lambda_{4}=U, \lambda_{5}=U+c .
$$

The 1- and 5-fields are genuinely non linear [45], and the 2-3- 4-field is linearly degenerate, since:

$$
\nabla_{W} \lambda_{2}(W) \cdot r_{2}(W)=\nabla_{W} \lambda_{3}(W) \cdot r_{3}(W)=\nabla_{W} \lambda_{4}(W) \cdot r_{4}(W)=0
$$

where $r_{k}$ stand for the five right eigenvectors of the Jacobian matrix $\frac{\partial F}{\partial W}$. Whatever the EOS is, both the pressure and the velocity are Riemann invariants in the three linearly degenerate fields. Jump conditions simply write ( $\sigma$ stands for the speed of the discontinuity between left state indexed by $a$ and right state indexed by $b)$ :

$$
-\sigma[W]_{a}^{b}+[F(W)]_{a}^{b}=0
$$

where $[\alpha]_{a}^{b}=\alpha_{b}-\alpha_{a}$. Using some basic algebra, one gets the following counterpart:

$$
\begin{array}{rrr}
v=U-\sigma, & \rho v\left[\left(e+\frac{P}{\rho}+\frac{v^{2}}{2}\right)\right]_{a}^{b} & =0, \\
\rho v[v]_{a}^{b}+[P]_{a}^{b}=0, & \rho v[C]_{a}^{b}=0, \\
{[\rho v]_{a}^{b}=0,} & \rho v[\psi]_{a}^{b}=0 .
\end{array}
$$

We also briefly recall that the list of Riemann invariants in the 1-rarefaction wave (respectively the 5-rarefaction wave) are $I_{1}=\left\{s, U+\int_{0}^{\rho}(c(\rho, s, C, \psi) / \rho) \mathrm{d} \rho, \psi, C\right\}$ (respectively $\left.I_{5}=\left\{s, U-\int_{0}^{\rho}(c(\rho, s, C, \psi) / \rho) \mathrm{d} \rho, \psi, C\right\}\right)$. Details on computation of specific entropy are recalled in Appendix B of [19]. Note also that: $I_{2,3,4}=\{P, U\}$. 


\section{Equation of state}

The next sections are dedicated to EOS which are such that the internal energy may be expressed in terms of some analytic function of the unknowns. The specific case where thermodynamical coefficients issue from tabulated laws will be discussed in Section 7.4.

We now introduce three distinct classes of EOS. The first one, which is noted $T_{1}$, contains EOS which agree with:

$$
\rho e=\phi_{1}(P, \rho, C, \psi)=\rho\left(a_{1}(P)+b_{1}(P) C+c_{1}(P) \psi\right)+d_{1}(P) .
$$

The second class contains EOS which do not lie in $T_{1}$ but nevertheless agree with:

$$
\rho e=\phi_{2}(P, C, \psi)=f_{2}(C, \psi) h_{2}(P)+g_{2}(C, \psi)
$$

where both $f_{2}$ and $g_{2}$ should differ from constants. The third class $T_{3}$ contains the remaining.

Note first that for given pressure $P=P_{\text {ref }}$, the function $\phi_{1}\left(P_{\text {ref }}, \rho, C, \psi\right)$ is linear w.r.t. unknowns $\rho, \rho C$ and $\rho \psi$. This has important consequences as will be discussed later. Note for instance that Tamman EOS and single component perfect gas EOS belong to the first class. It also includes EOS such as Tait EOS for solid material (see for instance [28]), since this one reads:

$$
\rho e=\frac{P}{\gamma-1}+\frac{\gamma(B-A)}{\gamma-1}
$$

where $A, B$ and $\gamma$ are positive constants.

The second class contains laws such as the stiffened gas EOS [40-42]

$$
\rho e=\frac{P-P_{\infty}(\psi)}{\gamma(\psi)-1}
$$

and the mixture of perfect gases [1]

$$
\rho e=\frac{P}{\gamma(C)-1}
$$

Note of course that Van der Waals EOS [35]:

$$
\begin{gathered}
\rho e=\rho C_{v} T-a(\rho)^{2}, \\
\left(P+a(\rho)^{2}\right)(1-b \rho)=\rho R T
\end{gathered}
$$

does not belong to the first or second class, nor does Mie-Gruneisen EOS (unless of course in some degenerated cases where they identify with previous mentioned laws, given specific (say null) values of constants imbeded). Obviously complex laws such as those described in [37] and in [32] are in $T_{3}$.

\section{Properties of Godunov type schemes with any EOS}

All results in the present section are independent of the kind of EOS application.

Let $\Phi$ be a regular function from $\mathbb{R}^{5}$ to $\mathbb{R}^{5}$ defined by (6) and $\Psi$ its inverse (we use the notation $Y=\Phi(W)$ ). For practical applications, we either use $g(\rho, s)=1 / \rho$ (see $[10,11]$ ), or $g(\rho, s)=\rho$ - in that case, the scheme is close to PVRS scheme proposed by Toro [46]-, or $g(\rho, s)=s$ in order to cope with vacuum [20]. Recall that 
the variable $Y^{t}=(U, P, s, C, \psi)$ enables to symmetrize the system. A detailed comparison of performances of VFRoe-ncv scheme with other well-known schemes is available in [18]. Schemes used herein take the form:

$$
h_{i}\left(W_{i}^{n+1}-W_{i}^{n}\right)+\delta t\left(F\left(\Psi\left(Y_{i+1 / 2}^{*}\right)\right)-F\left(\Psi\left(Y_{i-1 / 2}^{*}\right)\right)\right)=0
$$

where $h_{i}$ and $\delta t$ respectively denote the mesh size and the time step chosen in agreement with a CFL condition, $W_{i}^{n}$ stands for the mean value of conservative variable $W$ over cell $i$ at time $t_{n}$, and $Y_{i+1 / 2}^{*}$ is the exact (or approximate) value of the associated Riemann problem at the interface between two neighbouring cells with associated cell values $W_{i}^{n}$ and $W_{i+1}^{n}$. This provides updated value of conservative variable $W_{i}^{n+1}$, which enables to get the natural "obvious" definition of $e_{i}^{n}$ :

$$
\rho_{i}^{n} e_{i}^{n}=E_{i}^{n}-\frac{1}{2} \rho_{i}^{n} U_{i}^{n} U_{i}^{n}
$$

and standard definitions: $U_{i}^{n}=Q_{i}^{n} / \rho_{i}^{n}, C_{i}^{n}=(\rho C)_{i}^{n} / \rho_{i}^{n}$, (and if required $\left.\psi_{i}^{n}=(\rho \psi)_{i}^{n} / \rho_{i}^{n}\right)$. Hence, one may then extract $P_{i}^{n}$ as the value of the function $P$ (given by the EOS (5)) for given arguments $\rho_{i}^{n}, e_{i}^{n}, C_{i}^{n}, \psi_{i}^{n}$, and we set here:

$$
p_{i}^{n}=P_{i}^{n} .
$$

It is emphasized here that this "natural" definition of $p_{i}^{n}$ will be modified in Section 6 which deals with EOS in $T_{2} \cup T_{3}$. We recall that due to the specific form of the governing equations, both $\mathrm{C}$ and $\psi$ are Riemann invariants through the 1-field and the 5 -field. We introduce intermediate states indexed $l, r$ on the left and right side of the contact discontinuity, and index $L, R$ the left and right initial condition in the Riemann problem. If we assume that the genuinely non linear fields contain some discontinuity, we still have: $[C]_{L}^{l}=[\psi]_{L}^{l}=[C]_{r}^{R}=[\psi]_{r}^{R}=0$. Furthermore:

Property 1. Assume that we use either the exact Godunov scheme or some approximate Godunov scheme such as VFRoe-ncv scheme (see appendix, or [11, 18, 20]) in terms of $Y^{t}=(U, P, g(\rho, s), C, \psi)$. Intermediate states indexed $Y_{l}$ and $Y_{r}$ agree with:

$$
\begin{array}{cl}
C_{L}=C_{l}, & C_{r}=C_{R} \\
\psi_{L}=\psi_{l}, & \psi_{r}=\psi_{R}, \\
U_{l}=U_{r}, & P_{l}=P_{r}
\end{array}
$$

given left and right initial states $Y_{L}=\Phi\left(W_{L}\right)$ and $Y_{R}=\Phi\left(W_{R}\right)$.

The proof is straightforward for Godunov scheme, and very easy for VFRoe-ncv scheme (see [18]). On this basis, we also obviously check that for both solvers mentioned above, the following holds:

Property 2. Assume that the initial condition of a Riemann problem fulfills: $U_{L}=U_{R}$ and $P_{L}=P_{R}$, then, intermediate states in Godunov scheme and VFRoe-ncv scheme with variable $Y$ given by (6) agree with:

$$
\begin{aligned}
& U_{l}=U_{r}=U_{L}=U_{R}, \\
& P_{l}=P_{r}=P_{L}=P_{R} .
\end{aligned}
$$

The proof is well known for Godunov scheme, and straightforward for the VFRoe-ncv scheme with variable $Y$ given by (6).

Property 3. For given initial data in agreement with: $U_{k}^{n}=U_{0}$ and $p_{k}^{n}=P_{0}$ for $k=i-1, i, i+1$, Godunov scheme and VFRoe-ncv scheme with variable $Y$ given by $(6)$ ensure that: $U_{i}^{n+1}=U_{0}$. 

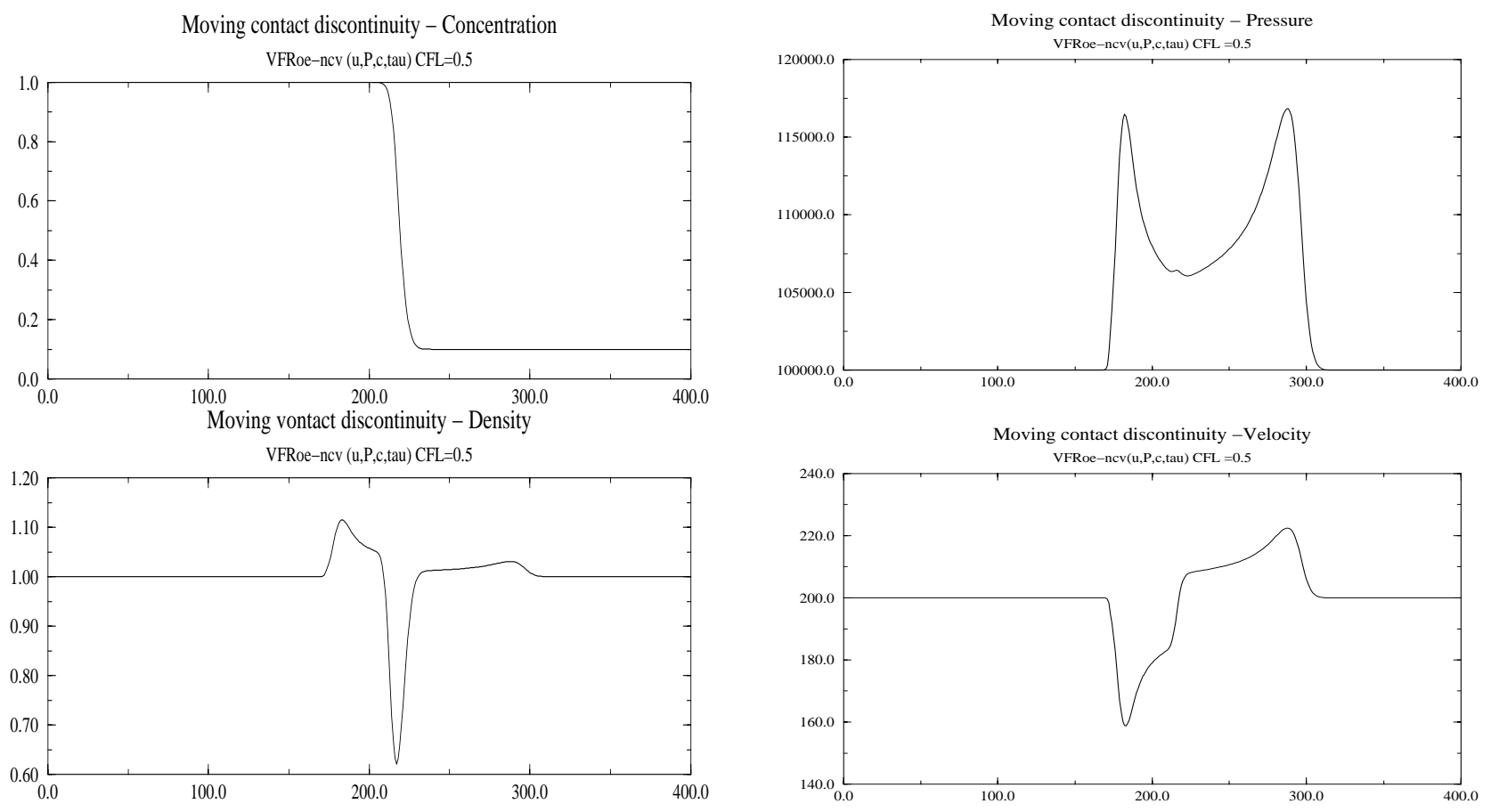

FIGURE 2. Moving contact discontinuity on coarse mesh.

\section{Behaviour of Godunov type schemes with EOS in $T_{1}$}

In addition to Property 3, we have:

Property 4. For given EOS in $T_{1}$, and for given initial data in agreement with: $U_{k}^{n}=U_{0}$ and $p_{k}^{n}=P_{0}$ for $k=i-1, i, i+1$, Godunov scheme and VFRoe-ncv scheme with variable $Y$ given by (6) also ensure that:

$$
p_{i}^{n+1}=P\left(\rho_{i}^{n+1}, e_{i}^{n+1}, C_{i}^{n+1}, \psi_{i}^{n+1}\right)=P_{0}
$$

Thus these schemes perfectly preserve unsteady contact discontinuities when restricting to EOS in $T_{1}$.

Note that $p_{i}^{n+1}=P\left(\rho_{i}^{n+1}, e_{i}^{n+1}, C_{i}^{n+1}, \psi_{i}^{n+1}\right) \neq P_{0}$ when the EOS does not lie in $T_{1}$; in that case, $U_{i}^{n+2}$ no longer remains equal to $U_{0}$.

\section{Behaviour of Godunov type schemes with EOS in $T_{2}$ OR $T_{3}$}

If we still use previous definition $p_{i}^{n+1}=P_{i}^{n+1}$, where $P_{i}^{n+1}=P\left(\rho_{i}^{n+1}, e_{i}^{n+1}, C_{i}^{n+1}, \psi_{i}^{n+1}\right)$, as defined in (5), Property 4 mentioned above is violated here. We first give some results obtained using EOS in $T_{2}$ as follows: $\rho e=P /(\gamma(C)-1)$ where $\gamma(C)=1,4 C+5,5(1-C)$. This corresponds to some stiffened gas EOS (with $\left.P_{\infty}=0\right)$. Initial conditions are such that both $U$ and $P$ should remain constant w.r.t. time and space. Results presented below (Figs. 2 and 3) correspond to standard "first-order" VFRoe-ncv scheme, using CFL number 0.5, and regular meshes containing 400 nodes (coarse mesh though "fine" industrial mesh when considering the " 3 -D counterpart") and 40000 nodes (fine mesh). Note that the relative error in the $L^{\infty}$ norm is approximately $30 \%$ on the coarse mesh. The latter diminishes when refining the mesh, and is about $5 \%$ on the finest mesh. The numerical method nevertheless converges (in $L^{1}$ norm) towards the right solution when the mesh size is refined. We emphasize that there is no contradiction between Property 3 and behaviour of the velocity in Figures 2 and 3 (see remark after Property 4). 

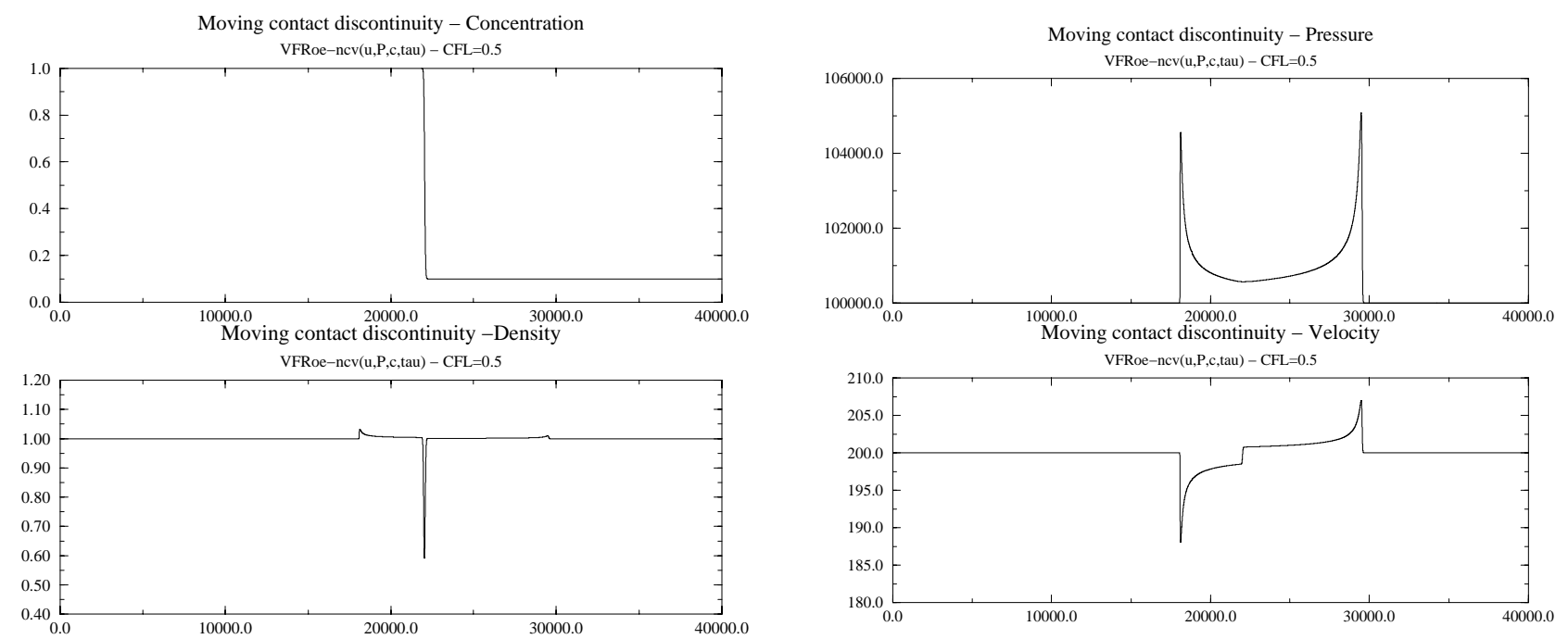

FiguRE 3. Moving contact discontinuity on fine mesh.

We turn now to EOS in $T_{3}$, focusing on Van der Waals EOS. Once more, Property 4 mentioned above is violated when using $P_{i}^{n}$ to initialize interface Riemann problems. We still emphasize that the basic first order conservative numerical method (exact Godunov or) VFRoe-ncv nonetheless provides convergent approximations of the solution. Figure 4 shows the behaviour of the error in the $L^{1}$ norm for both pressure and velocity variables, considering the first order scheme, with $C F L=0.5$, and uniform meshes with 200 cells up to 20000 cells. Initial conditions are:

$$
\begin{aligned}
& U_{L}=U_{R}=100, \rho_{L}=100, C_{L}=C_{R}=1 \\
& P_{L}=P_{R}=10^{6}, \rho_{R}=200, \psi_{L}=\psi_{R}=1 .
\end{aligned}
$$

The rate of convergence for $U, P$ is clearly $\frac{1}{2}$ as expected (since contact discontinuities are not perfectly preserved). However the very poor accuracy on coarse meshes is not appealing for industrial purposes.

\section{Hybrid Version of Godunov-Type schemes APPlied to $T_{2} \cup T_{3}$}

\subsection{Basic idea}

We now decompose any EOS in terms of EOS in $T_{1} \cup T_{2}$ and the remaining part, thus:

$$
\begin{aligned}
\phi_{3}(P, \rho, C, \psi) & =\rho e-\phi_{1}(P, \rho, C, \psi)-\phi_{2}(P, C, \psi), \\
\phi_{1}(P, \rho, C, \psi) & =\rho\left(a_{1}(P)+b_{1}(P) C+c_{1}(P) \psi\right)+d_{1}(P), \\
\phi_{2}(P, C, \psi) & =f_{2}(C, \psi) h_{2}(P)+g_{2}(C, \psi) .
\end{aligned}
$$

The decomposition should be achieved in order to "minimize" contributions in $T_{2} \cup T_{3}$. Hence, we define $a_{1}(P)$, $b_{1}(P), c_{1}(P), d_{1}(P)$ first, and then introduce $f_{2}(C, \psi), g_{2}(C, \psi)$ and $h_{2}(P)$ in order to "minimize" the residual part $\phi_{3}(P, \rho, C, \psi)$. This is achieved in practice in a natural way when focusing on analytic laws such as those imbeded in mixture of perfect gases, stiffened gas EOS, Van der Waals EOS, Chemkin database, Tamman EOS and many other laws such as those used to construct thermodynamical tables. For a given value of constant $P_{\text {ref }}$, we also introduce the function:

$$
g_{0}(C, \psi)=f_{2}(C, \psi) h_{2}\left(P_{\text {ref }}\right)+g_{2}(C, \psi) .
$$




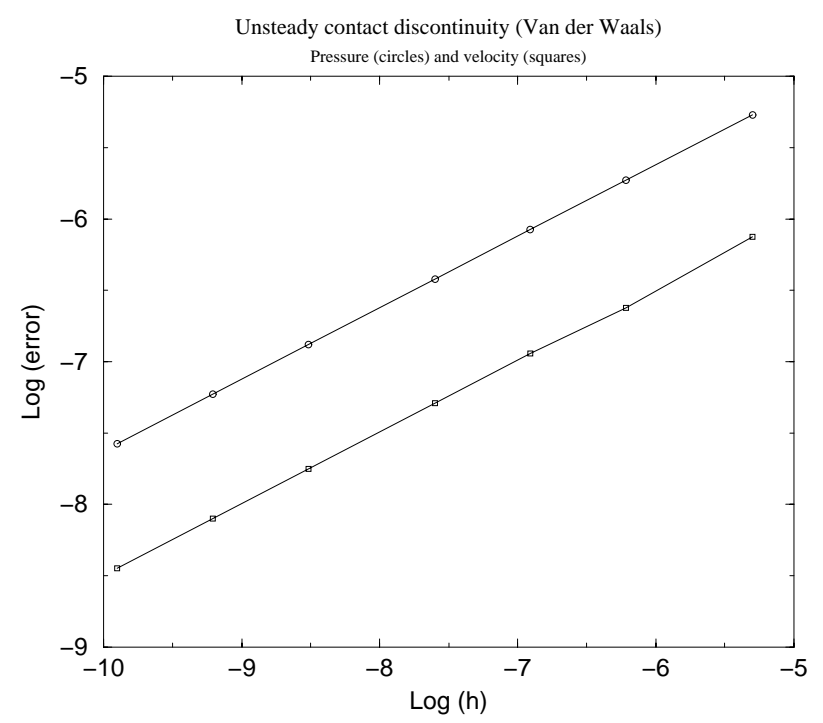

FiguRE 4. L1 error norm.

The latter quantity is governed by the following redundent equation when no discontinuity is present in the field:

$$
\frac{\partial g_{0}(C, \psi)}{\partial t}+U \frac{\partial g_{0}(C, \psi)}{\partial x}=0
$$

or alternatively by

$$
\frac{\partial \rho g_{0}(C, \psi)}{\partial t}+\frac{\partial\left(\rho g_{0}(C, \psi)\right) U}{\partial x}=0
$$

We note that this conservative formulation corresponds to additional jump relations:

$$
-\sigma\left[\rho g_{0}(C, \psi)\right]_{a}^{b}+\left[\rho g_{0}(C, \psi) U\right]_{a}^{b}=0
$$

When combined with the (true) jump relation associated with mass conservation this is equivalent to:

$$
\overline{\rho v}^{a b}\left[g_{0}(C, \psi)\right]_{a}^{b}=0 \text { and } \quad v=U-\sigma .
$$

where $\bar{\alpha}^{a b}=\left(\alpha_{a}+\alpha_{b}\right) / 2$. When $v$ is null (contact discontinuity), (25) is ensured of course. Besides, in genuinely non linear 1 and 5 fields, $\overline{\rho v}^{a b}$ is non zero but $g_{0}(C, \psi)$ is constant, hence $(25)$ holds, and therefore (24) is valid. We emphasize anyway that we will not use the "conservation law" for $\rho g_{0}(C, \psi)$, since it does not correspond to any physically conserved quantity, even if the EOS is exactly in $T_{2}$. Moreover, Abgrall's analysis has confirmed that this quantity is not the adequate variable to propagate.

For regular solutions of the basic five equation model, the redundent governing equation for $\phi_{3}$ is:

$$
\frac{\partial}{\partial t} \phi_{3}(P, \rho, C, \psi)+U \frac{\partial}{\partial x} \phi_{3}(P, \rho, C, \psi)+\left(\hat{\gamma} P \frac{\partial \phi_{3}}{\partial P}+\rho \frac{\partial \phi_{3}}{\partial \rho}\right) \frac{\partial U}{\partial x}=0
$$

which of course may degenerate if $\phi_{3}=0$. Unlike when dealing with EOS in $T_{2}$, one cannot provide a conservative re-formulation of (26) which enables to retrieve the true jump conditions. We may thus expect some greater difficulties when attempting to compute the extra non conservative governing equation for $\phi_{3}$ [24]. 
Focus for instance on Van der Waals EOS, then:

$$
\left\{\begin{aligned}
\rho e & =\phi_{1}(P, \rho, C, \psi)+\phi_{2}(P, C, \psi)+\phi_{3}(P, \rho, C, \psi) \\
\phi_{1}(P, \rho, C, \psi) & =\frac{(1-b \rho) P}{\gamma-1} \\
\phi_{2}(P, C, \psi) & =0 \\
\phi_{3}(P, \rho, C, \psi) & =a \rho^{2}\left(\frac{-b \rho}{\gamma-1}+\frac{2-\gamma}{\gamma-1}\right)
\end{aligned}\right.
$$

Obviously in this particular case, the function $g_{0}$ is null.

\subsection{Numerical scheme}

The basic scheme is the following for any EOS:

$$
\begin{aligned}
& h_{i}\left(W_{i}^{n+1}-W_{i}^{n}\right)+\delta t\left(F\left(\Psi\left(Y_{i+1 / 2}^{*}\right)\right)-F\left(\Psi\left(Y_{i-1 / 2}^{*}\right)\right)\right)=0, \\
& h_{i}\left(\left(g_{0}\right)_{i}^{n+1}-\left(g_{0}\right)_{i}^{n}\right)+\delta t \hat{U}_{i}\left(\left(g_{0}\right)_{i+1 / 2}^{*}-\left(g_{0}\right)_{i-1 / 2}^{*}\right)=0, \\
& h_{i}\left(\left(\phi_{3}\right)_{i}^{n+1}-\left(\phi_{3}\right)_{i}^{n}\right)+\delta t \hat{U}_{i}\left(\left(\phi_{3}\right)_{i+1 / 2}^{*}-\left(\phi_{3}\right)_{i-1 / 2}^{*}\right)+\delta t \hat{H}_{i}\left(U_{i+1 / 2}^{*}-U_{i-1 / 2}^{*}\right)=0,
\end{aligned}
$$

with

$$
\begin{aligned}
2 \hat{U}_{i} & =U_{i+1 / 2}^{*}+U_{i-1 / 2}^{*}, \\
2 \hat{H}_{i} & =\left(\hat{\gamma} P \frac{\partial \phi_{3}}{\partial P}+\rho \frac{\partial \phi_{3}}{\partial \rho}\right)_{i-1 / 2}^{*}+\left(\hat{\gamma} P \frac{\partial \phi_{3}}{\partial P}+\rho \frac{\partial \phi_{3}}{\partial \rho}\right)_{i+1 / 2}^{*}
\end{aligned}
$$

The definition of the numerical flux is the following:

$$
F\left(W^{*}\right)=\left(\rho^{*} U^{*}, \rho^{*} U^{*} C^{*}, \rho^{*} U^{*} U^{*}+P^{*}, U^{*}\left(\frac{\rho^{*}\left(U^{*}\right)^{2}}{2}+P^{*}\right)+U^{*}(\rho e)^{*}, \rho^{*} U^{*} \psi^{*}\right)
$$

where $(\rho e)^{*}=\phi_{1}\left(P^{*}, \rho^{*}, C^{*}, \psi^{*}\right)+\phi_{2}\left(P^{*}, C^{*}, \psi^{*}\right)+\phi_{3}\left(P^{*}, \rho^{*}, C^{*}, \psi^{*}\right), W^{*}=\Psi\left(Y^{*}\right)$ and $\left(g_{0}\right)^{*}=g_{0}\left(C^{*}, \psi^{*}\right)$. The series $\left(f_{2}\right)_{i}^{k}$ and $\left(g_{2}\right)_{i}^{k}$ issue from the computation of $g_{0}$ setting $h_{2}\left(P_{\text {ref }}\right)=0$ and $h_{2}\left(P_{\text {ref }}\right)=1$. They should not be confused with $f_{2}\left(C_{i}^{k}, \psi_{i}^{k}\right)$ and $g_{2}\left(C_{i}^{k}, \psi_{i}^{k}\right)$.

The cell pressure used to compute the local Riemann problems at the beginning of the next time step namely:

$$
p_{i}^{n+1}=\tilde{P}_{i}^{n+1}
$$

is obtained by solving the following problem:

$$
\| \begin{aligned}
& \text { Find } \tilde{P}_{i}^{n+1} \text { solution of } \\
& \rho_{i}^{n+1} e_{i}^{n+1}-\left(\left(g_{2}\right)_{i}^{n+1}+\left(\phi_{3}\right)_{i}^{n+1}\right)=\left(f_{2}\right)_{i}^{n+1} h_{2}\left(\tilde{P}_{i}^{n+1}\right)+\phi_{1}\left(\tilde{P}_{i}^{n+1}, \rho_{i}^{n+1}, C_{i}^{n+1}, \psi_{i}^{n+1}\right)
\end{aligned}
$$

where $\rho_{i}^{n+1} e_{i}^{n+1}=E_{i}^{n+1}-\left(\left(Q_{i}^{n+1}\right)^{2}\right) /\left(2 \rho_{i}^{n+1}\right)$ and with given values $E_{i}^{n+1}, Q_{i}^{n+1}, \rho_{i}^{n+1}, C_{i}^{n+1}, \psi_{i}^{n+1}$ provided by discrete conservative equations, and $\left(f_{2}\right)_{i}^{n+1},\left(g_{2}\right)_{i}^{n+1},\left(\phi_{3}\right)_{i}^{n+1}$ provided by discrete non-conservative equations. Note that (28) implies that $\phi_{1}$ or $h_{2}$ are non zero. 


\subsection{Main property and remarks}

We now have the main property:

Property 5. For any EOS in $T_{1} \cup T_{2} \cup T_{3}$, and for given initial data in agreement with: $U_{k}^{n}=U_{0}$ and $p_{k}^{n}=P_{0}$ for $k=i-1, i, i+1$, the above mentioned scheme (27) ensures that:

$$
p_{i}^{n+1}=P_{0} \quad \text { and } \quad U_{i}^{n+1}=U_{0}
$$

when using Godunov scheme and VFRoe-ncv scheme with variable $Y$ given by (6).

6.3.1. Main remarks

Remark 1. We first note that the frame of EOS which lie exactly in $T_{1}$ is contained in the global formulation above since in that case, both $\phi_{2}$ and $\phi_{3}$ are null, and as a result $P_{i}^{n+1}$ is computed as:

$$
\| \begin{aligned}
& \text { Find } P_{i}^{n+1} \text { solution of } \\
& \rho_{i}^{n+1} e_{i}^{n+1}=\phi_{1}\left(P_{i}^{n+1}, \rho_{i}^{n+1}, C_{i}^{n+1}, \psi_{i}^{n+1}\right)
\end{aligned}
$$

and one retrieves the fully -standard-conservative scheme.

Remark 2. When considering the specific case of stiffened gas EOS, it is emphasized that the proposed scheme (27) identifies with the proposal of Abgrall and Saurel [42], by setting $h_{2}(P)=P$ in $\phi_{2}(P, C, \psi)$.

Remark 3. From a numerical point of view, it is also necessary to point out that the numerical scheme which is used to compute governing equation of $\phi_{3}$ in (27) is consistent with conservative equations for total mass and mass species. This means that for given laws of the form:

$$
\phi_{3}(P, \rho, C, \psi)=\mu_{0} \rho+\mu_{1} \rho C+\mu_{2} \rho \psi
$$

the discrete equation of $\phi_{3}$ is exactly the counterpart of the linear combination of discrete equations of $\rho$ and $\rho C$. Though it would correspond to some "wrong" decomposition of the EOS - all these contributions should have been set in $T_{1}$-, one nonetheless needs to examine this "virtual" case. Thus, in that particular case, it may be rewritten in the form:

$$
\frac{\partial \phi_{3}(P, \rho, C, \psi)}{\partial t}+\frac{\partial U \phi_{3}(P, \rho, C, \psi)}{\partial x}=0
$$

from a continuous point of view, but one notices that the discrete governing equation of $\phi_{3}$ is also a linear combination of discrete equations of $\rho, \rho C, \rho \psi$, and thus retrieves the correct conservative form:

$$
\left.h_{i}\left(\left(\phi_{3}\right)_{i}^{n+1}-\left(\phi_{3}\right)_{i}^{n}\right)+\delta t\left(\left(U \phi_{3}\right)_{i+1 / 2}^{*}-\left(U \phi_{3}\right)_{i-1 / 2}^{*}\right)\right)=0
$$

The latter remark no longer holds when defining for instance $(\hat{H})_{i}=H_{i}^{n}$. Actually, some counterpart of this discretization $(\hat{H})_{i}=H_{i}^{n}$ has been experienced before to provide loss of stability in other computations (computation of Reynolds stress closures in compressible turbulent flows).

Remark 4. Actually, there is no proof whether the hybrid scheme (27) converges, and assuming it does, there is little evidence that it converges towards the right weak solution when discontinuities are present in the computational field, owing to the non conservative form of the whole scheme. This will be discussed in Section 7 . From an industrial point of view, it does not seem compulsory to get the right $(\hat{H})_{i}$, more precisely the one which yields correct jump conditions. This will be checked a posteriori when computing Van der Waals EOS which is a good example where the contribution in $T_{3}$ is not negligible when compared with the contribution in $T_{1}$. It nonetheless seems appealing from an academic point of view, but it must be underlined that feasibility in a one dimensional framework does not imply the counterpart in a three dimensional case. 


\subsubsection{Minor remarks}

Remark 5. We have implicitly assumed that all EOS will have some non zero contribution in at least one class among $T_{1}$ or $T_{2}$. Otherwise updating the cell pressure through relation (28) would be no longer feasible, and should be replaced by:

$$
\| \begin{aligned}
& \text { Find } \tilde{P}_{i}^{n+1} \text { solution of } \\
& \left(\phi_{3}\right)_{i}^{n+1}=\phi_{3}\left(\tilde{P}_{i}^{n+1}, \rho_{i}^{n+1}, C_{i}^{n+1}, \psi_{i}^{n+1}\right) .
\end{aligned}
$$

This frame is very unlikely to happen in practice, and all EOS considered herein which arise from the literature do have some contribution in $T_{1} \cup T_{2}$. This academic case will nonetheless be examined in Section 7.4.3.

Remark 6. We also note that formally, both second and third discrete equations in (27) might be put together. This is due to the fact that:

$$
\hat{\gamma} P \frac{\partial g_{0}}{\partial P}+\rho \frac{\partial g_{0}}{\partial \rho}=0
$$

and to the use of the superposition principle. We nonetheless will still distinguish both for at least two reasons. The continuous counterpart of the third discrete equation in (27) cannot be recast in true conservative form, whereas this may be achieved for the second equation in (27) which governs any function $\alpha(C, \psi)$, as discussed above. Second, we note that doing so (i.e. gathering both contributions) would result in an illposedness of the value $P_{i}^{n+1}$ when precisely focusing on EOS in $T_{2}$. Last but not least, we will check that the accuracy on very fine meshes may be slowed down when doing so (see Sect. 7.4 about the influence of the decomposition).

\section{NuMERICAL RESULTS}

\subsection{Stiffened gas EOS}

Numerical results below are dedicated to simplified stiffened gas EOS in $T_{2}\left(\right.$ since $\left.\left(P_{\infty}\right)_{1}=\left(P_{\infty}\right)_{2}=0\right)$ as follows:

$$
\rho e(P, \rho, C, \psi)=\frac{P}{\gamma(\psi)-1}
$$

where $\gamma(\psi)=1.667 \psi+1.4(1-\psi)$. The decomposition is thus the following:

$$
\left\{\begin{array}{l}
\rho e=\phi_{2}(P, C, \psi)=f_{2}(C, \psi) h_{2}(P)+g_{2}(C, \psi), \\
h_{2}(P)=P, f_{2}(C, \psi)=\frac{1}{\gamma(\psi)-1}, g_{2}(C, \psi)=\frac{P_{\infty}(\psi)}{\gamma(\psi)-1}, \\
\left(\phi_{1}(P, \rho, C, \psi)=\phi_{3}(P, \rho, C, \psi)=0\right) .
\end{array}\right.
$$

A first series of results corresponds to initial conditions proposed by Sandra Rouy [40]:

$$
\begin{aligned}
& U_{L}=0, P_{L}=120000, \rho_{L}=0.192, C_{L}=1, \psi_{L}=1, \\
& U_{R}=0, P_{R}=100000, \rho_{R}=1.156, C_{R}=1, \psi_{R}=0 .
\end{aligned}
$$

Results presented below (Fig. 5) correspond to standard "first-order" VFRoe-ncv scheme, using CFL number 0.5, and regular meshes containing 100 nodes (coarse mesh), and 40000 nodes (fine mesh). Results obtained with the hybrid version of the approximate Godunov scheme apparently converge towards the entropy solution when the mesh is refined. Nonetheless, the approximate solution on coarse mesh is indeed nicer when using the hybrid version (27). 

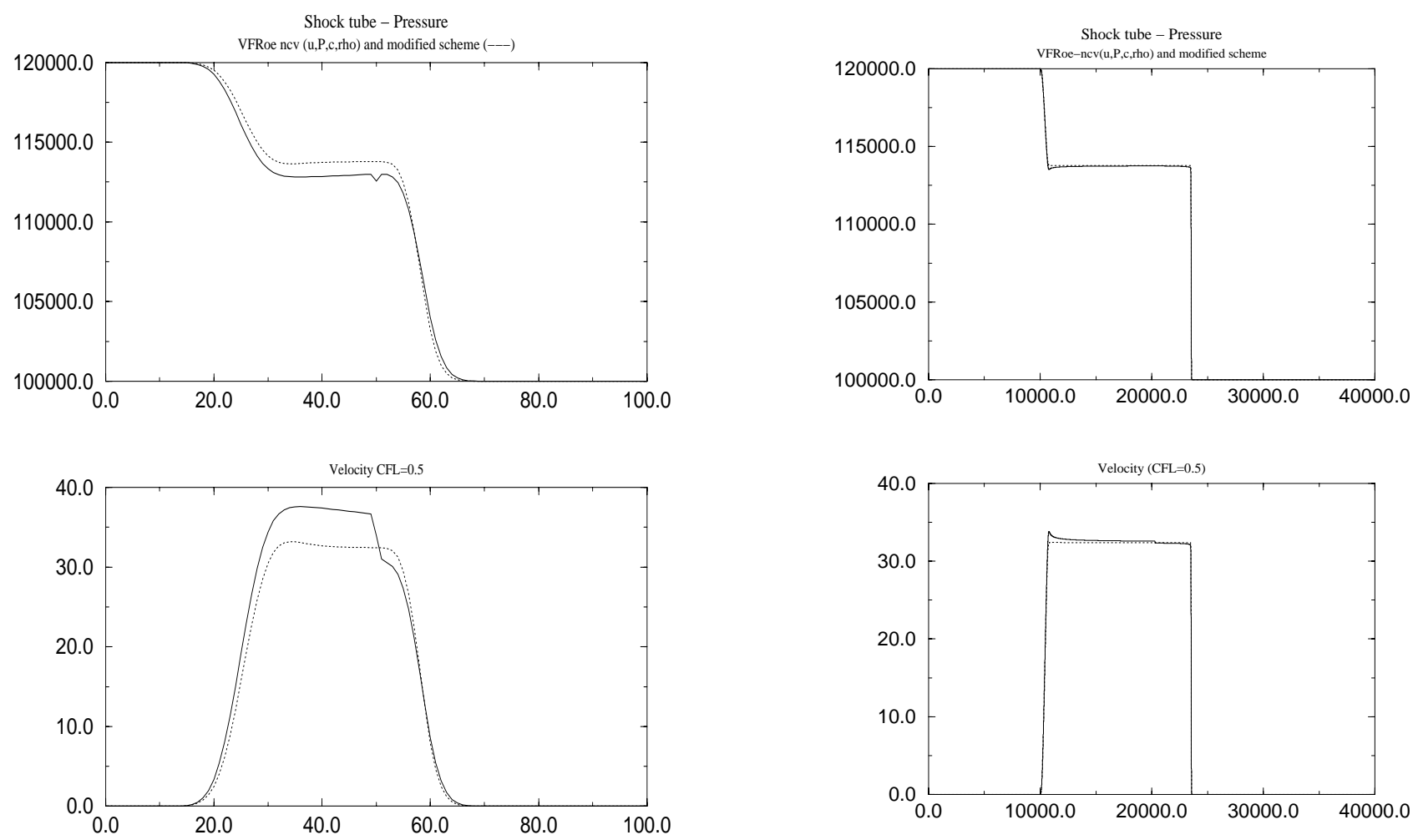

FiguRE 5. Shock tube with EOS in $T_{2}$ - coarse mesh (left), fine mesh (right).

We turn now to a simpler set of initial condition, as follows:

$$
\begin{aligned}
& U_{L}=\left(\left(\frac{1}{\rho_{R}}-\frac{1}{\rho_{2}}\right)\left(P_{L}-P_{R}\right)\right)^{0.5}, P_{L}=P_{R} \frac{\beta_{R} z-1}{\beta_{R}-z}, \rho_{L}=4.0, \psi_{L}=1, C_{L}=1 \\
& U_{R}=0, \quad P_{R}=100000, \quad \rho_{R}=1.0, \psi_{R}=0, C_{R}=1 \text {, }
\end{aligned}
$$

where $\beta_{R}=\frac{\gamma_{2}+1}{\gamma_{2}-1}$, and $z=\frac{\rho_{2}}{\rho_{R}}$ with $\rho_{2}=2$. This results in a pure right going 3 shock. This Riemann problem is close to the previous one, since the difference lies in the ghost 1-wave here, which turned to be a rarefaction wave before. However, one may clearly expect that this regular wave cannot inhibit the convergence towards the right solution. In addition, the present case enables to get rid of the compulsory error in the prediction of the regular 1-rarefaction wave, which might hide some error in the computation of the 3-shock. In practice, the present initial conditions require that the hybrid scheme manages to compute the exact intermediate state of density on the right side of the (moving) contact discontinuity, which is not obvious at all. We have plot below the error using the $L^{1}$ norm. Uniform meshes contain from 100 up to 160000 cells. The $C F L$ number still equals 0.5. The error obviously vanishes as the mesh size tends towards zero (see Fig. 6). The rate of convergence for density is slightly greater than $\frac{1}{2}$, and the rate of convergence for $\mathrm{U}$ and $\mathrm{P}$ variables is 1 . We emphasize that the rate is $\frac{1}{2}$ for $\rho, U, P$ when using basic conservative scheme (Fig. 6).

\subsection{Van der Waals EOS}

Note that when restricting to Van der Waals EOS, there is no need to compute redundent information for (null) function $g_{0}$. Constants used in the EOS are: $a=1684.54, b=0.001692, R=461.5, C_{v}=1401.88$. We 


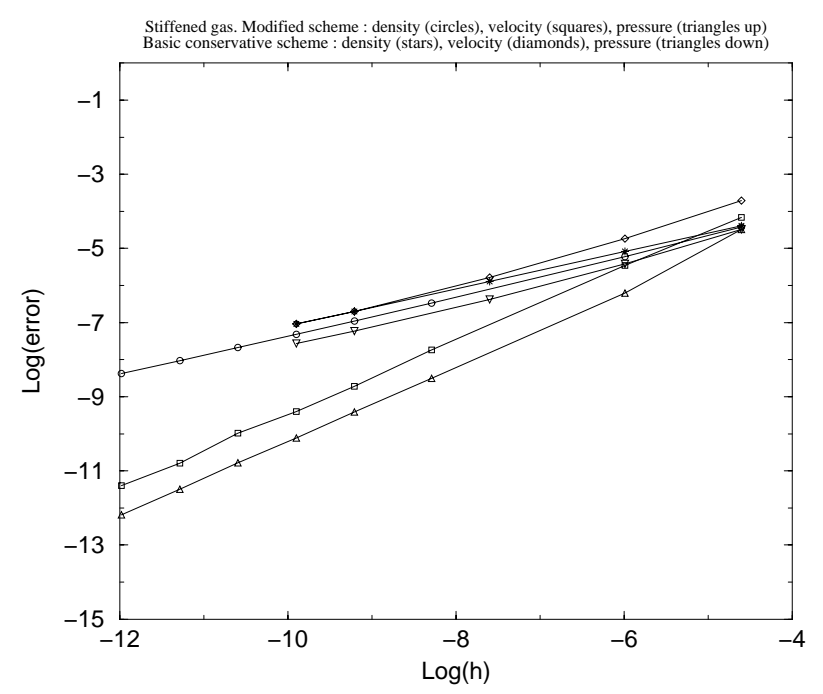

Figure 6. Pure unsteady 3 -shock with EOS in $T_{2}$ - error in $L^{1}$ norm.

recall below the decomposition:

$$
\left\{\begin{aligned}
\rho e & =\phi_{1}(P, \rho, C, \psi)+\phi_{2}(P, C, \psi)+\phi_{3}(P, \rho, C, \psi) \\
\phi_{1}(P, \rho, C, \psi) & =\frac{(1-b \rho) P}{\gamma-1} \\
\phi_{2}(P, C, \psi) & =0 \\
\phi_{3}(P, \rho, C, \psi) & =a \rho^{2}\left(\frac{-b \rho}{\gamma-1}+\frac{2-\gamma}{\gamma-1}\right)
\end{aligned}\right.
$$

\subsubsection{Shock tube case}

We focus here on test case proposed by Letellier and Forestier [35]. Initial data is given by [35]:

$$
\begin{aligned}
& U_{L}=0, P_{L}=37311358, \rho_{L}=333, C_{L}=1, \psi_{L}=1, \\
& U_{R}=0, P_{R}=21770768, \rho_{R}=111, C_{R}=1, \psi_{R}=1 .
\end{aligned}
$$

Figures 7 and 8 refer to the comparison of both approximations provided by the basic fully conservative scheme and the hybrid scheme when computing a shock tube case on different meshes. Results are obviously more appealing when using the hybrid version of the scheme. The error in $L^{1}$ norm associated with the hybrid scheme is given on the last Figure 9, as a function of the mesh size. We note that on the finest mesh which is clearly out of reach of present computers for 3D calculations, the decrease of error slows down.

For seak of completeness, we now examine the remaining two configurations of the basic 1D Riemann problem, which either involve two shock waves or two rarefactions waves.

\subsubsection{Double rarefaction wave}

We now examine some symmetrical double rarefaction wave. This enables to predict the behaviour of the scheme close to the wall boundary behind some bluff body, when applying for the mirror technique (see [11]). Initial conditions are now:

$$
\begin{aligned}
& U_{L}=-100, P_{L}=10^{7}, \rho_{L}=111, C_{L}=\psi_{L}=1 \\
& U_{R}=100, \quad P_{R}=10^{7}, \rho_{R}=111, C_{R}=\psi_{R}=1 .
\end{aligned}
$$



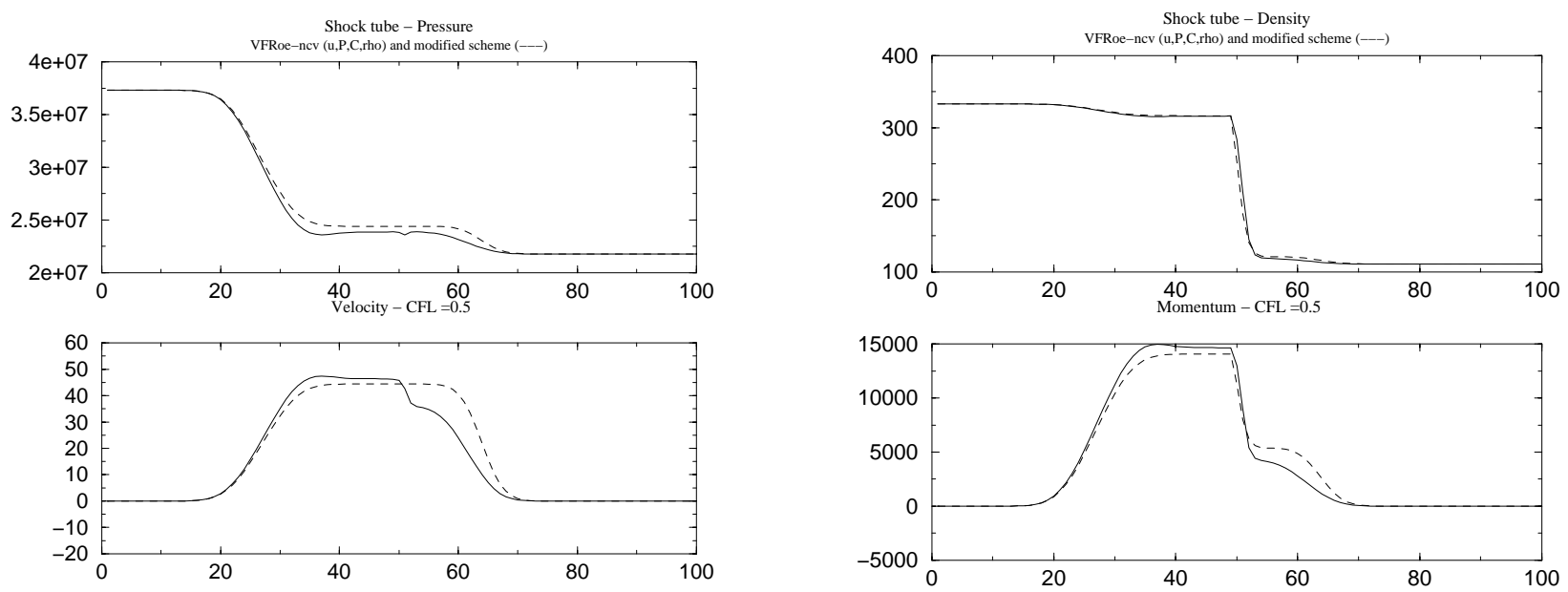

FIGURE 7 . Shock tube with EOS in $T_{3}-$ coarse mesh.
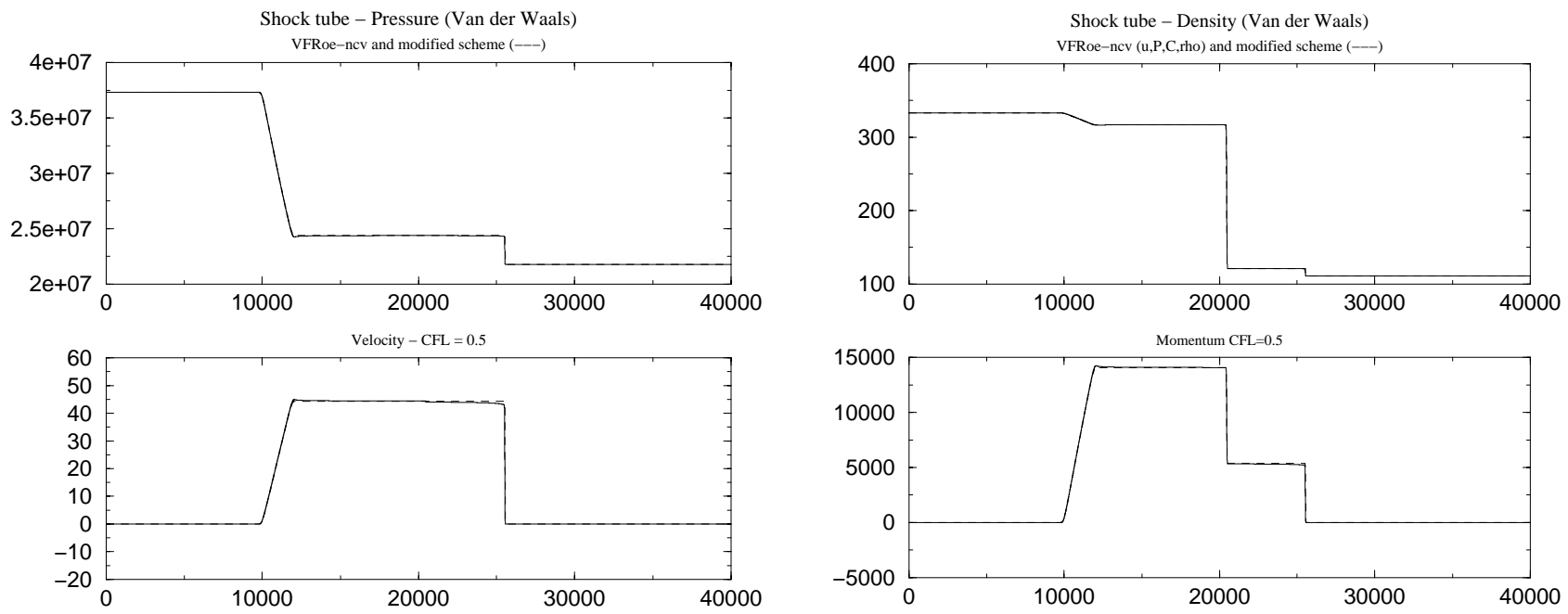

FiguRE 8. Shock tube with EOS in $T_{3}$ - finest mesh.

The time step is still in agreement with CFL condition, $C F L=0.5$. The mesh is composed of 200 regular cells. The first order version of the scheme has been used here (see Fig. 10-left). Note that the small glitch on the density at the initial position of the membrane is already present when using the standard Godunov scheme or VFRoe-ncv scheme in a fully conservative form. One might expect a rather nice behaviour of the scheme here since the exact solution contains no shock wave.

\subsubsection{Double shock wave}

Before going further on, we examine some symmetrical double shock wave. This provides an initial guess of what happens when the flow is impinging the wall boundary. Initial conditions are:

$$
\begin{aligned}
& U_{L}=100, \quad P_{L}=10^{7}, \rho_{L}=111, C_{L}=\psi_{L}=1 \\
& U_{R}=-100, P_{R}=10^{7}, \rho_{R}=111, C_{R}=\psi_{R}=1 .
\end{aligned}
$$

The CFL number is the same as above. The mesh still contains two hundred nodes (see Fig. 10-right). 


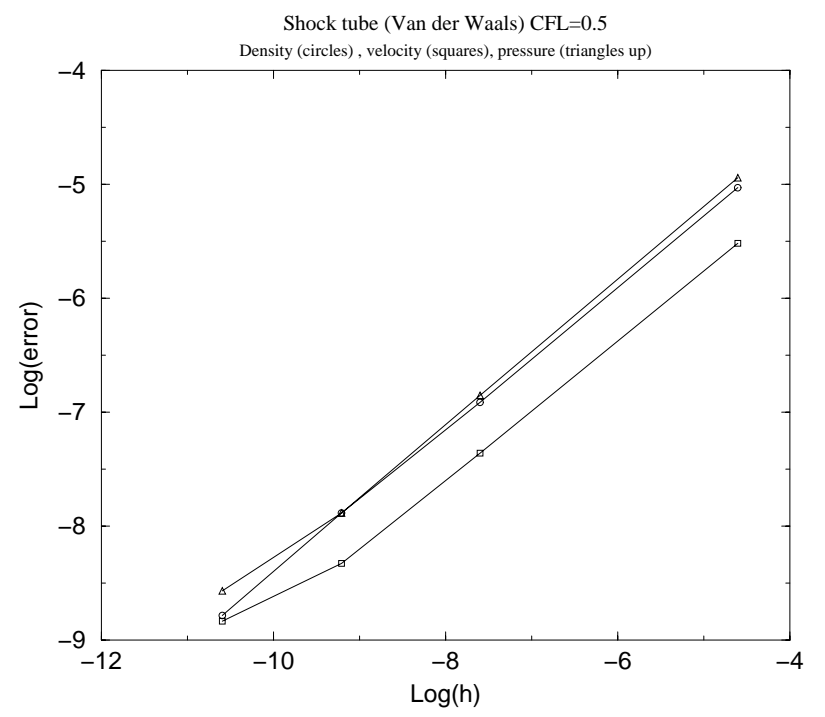

FiguRE 9. Error in $L^{1}$ norm for hybrid scheme.
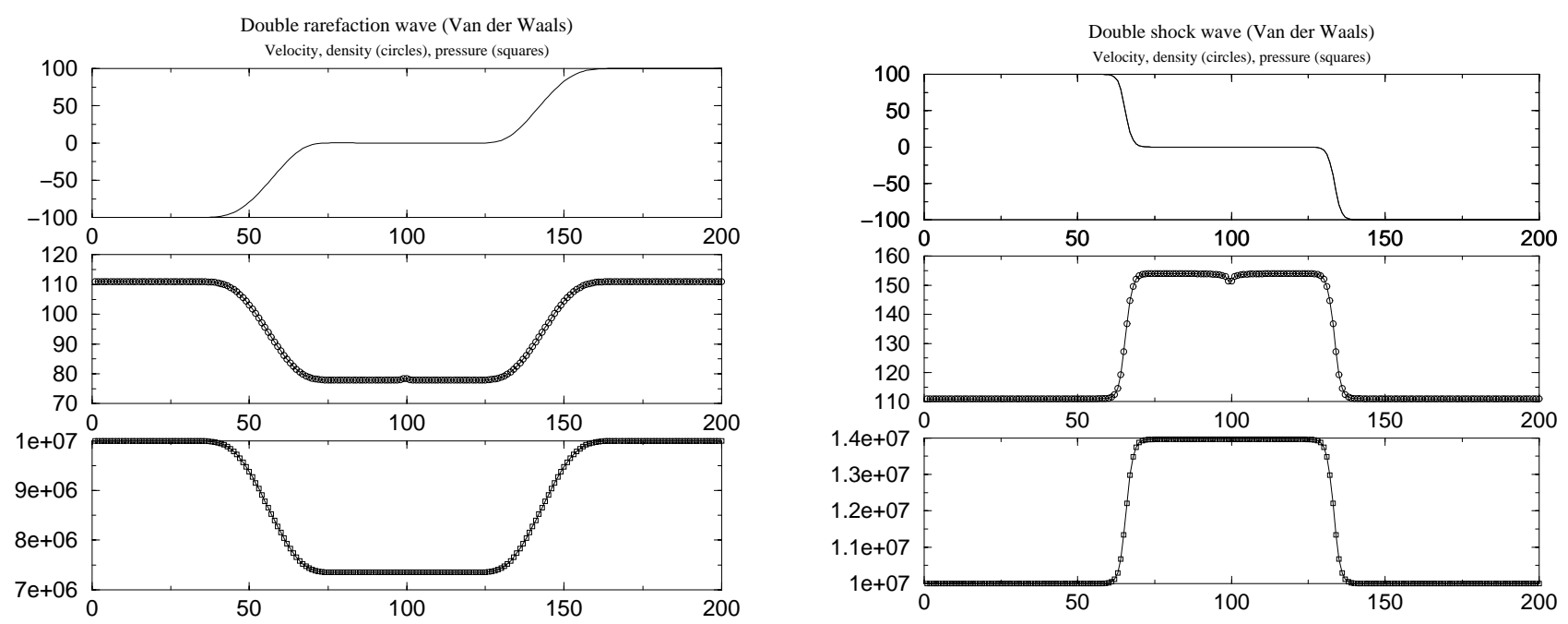

Figure 10. Double rarefaction wave (left) and double shock wave (right) with EOS in $T_{3}$.

\subsubsection{3-shock waves}

We now investigate some 3 -shock waves. Recall that one advantage here is that the 1-wave will be a "ghost" wave, and therefore will generate a much smaller amount of error, which might hide deficiencies occurring in shock waves when focusing on the standard shock tube apparatus. Hence, we first introduce initial conditions as follows:

$$
\begin{array}{ll}
U_{L}=U_{R}+\left(\left(\frac{1}{\rho_{R}}-\frac{1}{\rho_{2}}\right)\left(P_{L}-P_{R}\right)\right)^{0.5}, & \rho_{L}=4.0, C_{L}=\psi_{L}=1 \\
U_{R}=0, & \rho_{R}=1.0, C_{R}=\psi_{R}=1
\end{array}
$$


with $P_{R}=100000, \rho_{2}=2$ and $P_{L}>P_{R}$ solution of:

$$
2 \rho_{2} \rho_{R}\left(e\left(P_{L}, \rho_{2}\right)-e\left(P_{R}, \rho_{R}\right)\right)=\left(P_{L}+P_{R}\right)\left(\rho_{2}-\rho_{R}\right) .
$$

Intermediate states indexed 1,2 agree with $U_{L}=U_{1}=U_{2}, P_{L}=P_{1}=P_{2}, \rho_{L}=\rho_{1}$.

The error in $L^{1}$ norm is given in Figure 11. The finer mesh contains 160000 nodes and the coarser mesh 100 cells. In the range of sufficiently coarse meshes (from 100 up to 10000 cells), the error in $L^{1}$ norm for the density varies as $h^{1 / 2}$. We notice anyway, that the rate of convergence for both velocity and pressure is approximately 1 for meshes with 100 up to 10000 cells, but the error remains stationary (w.r.t. mesh size) for meshes containing more than ten thousand nodes. This obviously means that some -indeed small value- $O(1)$ error is present in the solution close to the 3 -shock wave. An ambiguous point is that it may only be exhibited when using mesh refinement which involves much more cells than one may afford in practice, and which is also seldomly investigated by developers. The counterpart in a $3 \mathrm{D}$ framework would require more than $10^{12}$ cells. This implies in practice that the hybrid scheme should not be disregarded. We will come back to similar comments below.

We turn now to different initial conditions where densities and pressures are much higher:

$$
\begin{aligned}
& U_{L}=U_{R}+\left(\left(\frac{1}{\rho_{R}}-\frac{1}{\rho_{2}}\right)\left(P_{L}-P_{R}\right)\right)^{0.5}, \rho_{L}=320.0, C_{L}=\psi_{L}=1, \\
& U_{R}=0, \quad \rho_{R}=80.0, \quad C_{R}=\psi_{R}=1,
\end{aligned}
$$

with $P_{R}=8000000, \rho_{2}=160$ and $P_{L}>P_{R}$ solution of:

$$
2 \rho_{2} \rho_{R}\left(e\left(P_{L}, \rho_{2}\right)-e\left(P_{R}, \rho_{R}\right)\right)=\left(P_{L}+P_{R}\right)\left(\rho_{2}-\rho_{R}\right) .
$$

We have plot here the error in $L^{1}$ norm in Figure 12. Similar comments as previous ones still hold here, and the rate of convergence for the conservative scheme is clearly $\frac{1}{2}$ for the density, the pressure and the velocity. This is due to the fact that the local amount of error around the contact discontinuity for pressure and velocity is so high that it inhibits rate 1 to be set. Once again, the error with the modified scheme becomes stationary when meshes involve more than $10^{4}$ cells.

Remark. In any case, it confirms that EOS in $T_{2}$ and EOS in $T_{3}$ should be distinguished, at least from a theoretical point of view. The occurrence of a true non conservative product $H(W) \partial_{x} U$ in the governing equation of $\phi_{3}$ inhibits the convergence towards the right solution, and this can only be seen on very fine meshes $\left(10^{5}\right.$ cells). These results are in agreement with scalar results obtained by Hou and Le Floch [24].

\subsection{Chemkin database}

We focus here on EOS provided in [32] and investigated in $[10,11]$. The internal energy is a polynomial function in terms of the local temperature $\mathrm{T}$.

$$
\left\{\begin{array}{l}
\rho e=r \mu_{0} \rho+\left(\mu_{1}-1\right) P+\sum_{2 \leq n \leq k} \mu_{n} \frac{P^{n}}{(r \rho)^{n-1}}, \\
P=r \rho T .
\end{array}\right.
$$

Straightforward decomposition yields:

$$
\left\{\begin{aligned}
\rho e & =\phi_{1}(\rho, P, C, \psi)+\phi_{2}(P, C, \psi)+\phi_{3}(\rho, P, C, \psi), \\
\phi_{1}(\rho, P, C, \psi) & =r \mu_{0} \rho+\left(\mu_{1}-1\right) P \\
\phi_{2}(P, C, \psi) & =0 \\
\phi_{3}(\rho, P, C, \psi) & =\sum_{2 \leq n \leq k} \mu_{n} \frac{P^{n}}{(r \rho)^{n-1}} .
\end{aligned}\right.
$$




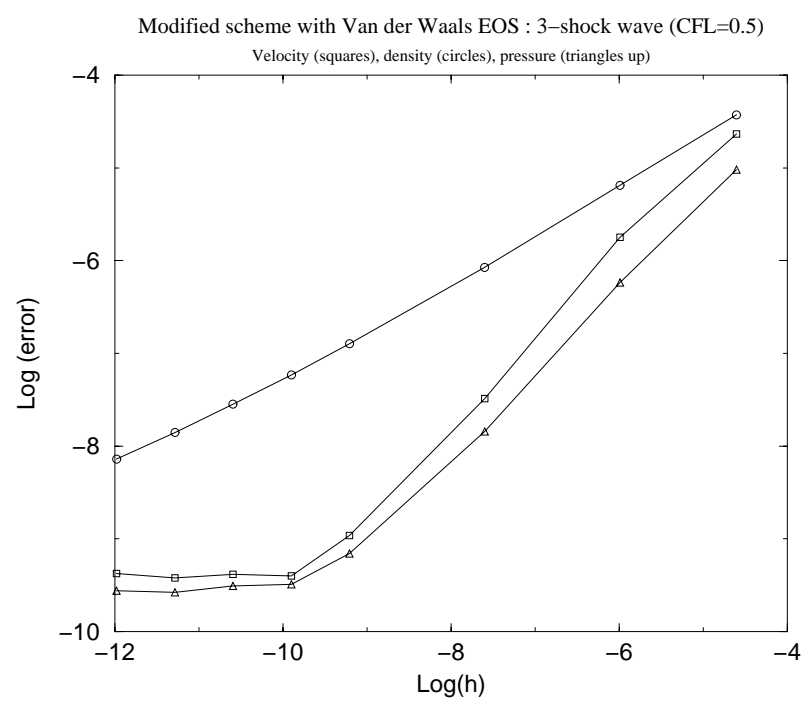

FiguRE 11. Error in $L^{1}$ norm for hybrid scheme.

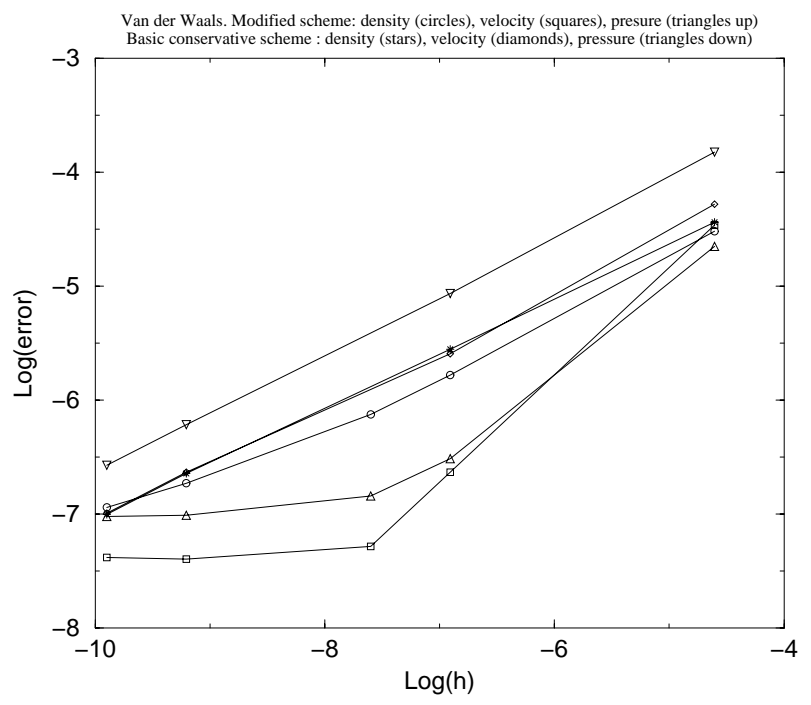

FiguRE 12. Error in $L^{1}$ norm for conservative and hybrid scheme.

We may simply compute the speed of acoustic waves as:

$$
c^{2}=\frac{\hat{\gamma} P}{\rho}=r T \frac{\mu_{1}+\sum_{2 \leq n \leq k} n \mu_{n} T^{n-1}}{\mu_{1}-1+\sum_{2 \leq n \leq k} n \mu_{n} T^{n-1}} .
$$

The whole algorithm only requires updating the cell pressure $p_{i}^{n+1}=\tilde{P}_{i}^{n+1}$ at the end of the time step as follows:

$$
\tilde{P}_{i}^{n+1}=\frac{(\rho e)_{i}^{n+1}-\mu_{0} r(\rho)_{i}^{n+1}-\left(\phi_{3}\right)_{i}^{n+1}}{\mu_{1}-1} .
$$

Remark. Note that unlike when using the basic Godunov or VFRoe-ncv schemes, this only requires an algebraic manipulation and does not require any Newton procedure to compute $P_{i}^{n+1}$ in each cell as a solution of:

$$
(\rho e)\left(P_{i}^{n+1}, \rho_{i}^{n+1}\right)=E_{i}^{n+1}-\frac{Q_{i}^{n+1} Q_{i}^{n+1}}{(2 \rho)_{i}^{n+1}}
$$

which results in a great decrease of the computational CPU time.

We refer to [11] which provides data of initial conditions used herein. The computations (Fig. 13) have been obtained using present approximate Godunov scheme VFRoe-ncv with $(\tau, U, P)$ variables. Other computations with help of Roe approximate Riemann solver are given in [12]. Details concerning entropy are briefly recalled in Appendix B of [19].

\subsection{Tabulated EOS}

For arbitrary non analytic EOS, we now define the decomposition of the EOS in the class $T_{1}$ and $T_{3}$. This may be achieved defining some function $d_{1}(P)=\frac{P}{\gamma_{1}-1}$, which is close enough to the real state law. The constant $\gamma_{1}$ 


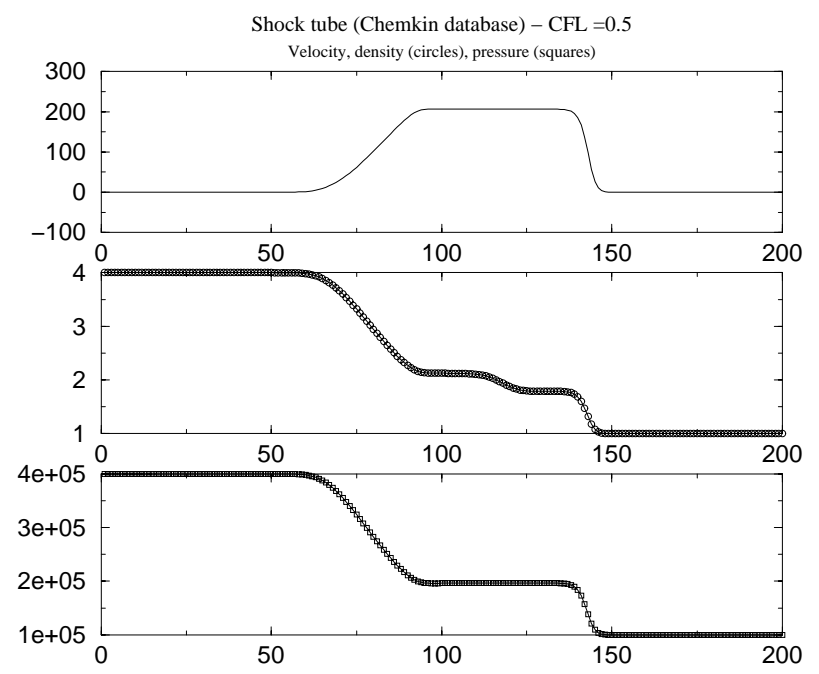

Figure 13. Shock tube using Chemkin database - coarse mesh.

is computed introducing some least square minimization process:

$$
\left\{\begin{aligned}
\phi_{1}(\rho, P, C, \psi) & =\frac{P}{\gamma_{1}-1} \\
\phi_{2}(P, C, \psi) & =0 \\
\phi_{3}(\rho, P, C, \psi) & =\rho e-\frac{P}{\gamma_{1}-1}
\end{aligned}\right.
$$

Thus the redundent equation which is computed reads:

$$
\frac{\partial}{\partial t} \phi_{3}(P, \rho, C, \psi)+U \frac{\partial}{\partial x} \phi_{3}(P, \rho, C, \psi)+\left(\rho e+P-\frac{\rho c^{2}}{\gamma_{1}-1}\right) \frac{\partial U}{\partial x}=0
$$

\subsubsection{Influence of decomposition}

We examine very briefly below whether some discrepancy in the decomposition implies some loss of accuracy, or in other words try to evaluate the stability of the overall method w.r.t. to the choice of the decomposition. Assume for instance that the real EOS reads $(\rho e)=\frac{P}{\gamma_{1}-1}$. Imagine that some - on purpose- error occurs in the process in such a way that the decomposition yields:

$$
\left\{\begin{aligned}
\phi_{1}(\rho, P, C, \psi) & =\frac{P}{\gamma_{2}-1} \\
\phi_{2}(P, C, \psi) & =0 \\
\phi_{3}(\rho, P, C, \psi) & =P\left(\frac{1}{\gamma_{1}-1}-\frac{1}{\gamma_{2}-1}\right)
\end{aligned}\right.
$$

where of course both constants are distinct. Despite from its simplicity, we first note that the resulting hybrid scheme does not compute the same approximation of the internal energy than the fully conservative scheme. 


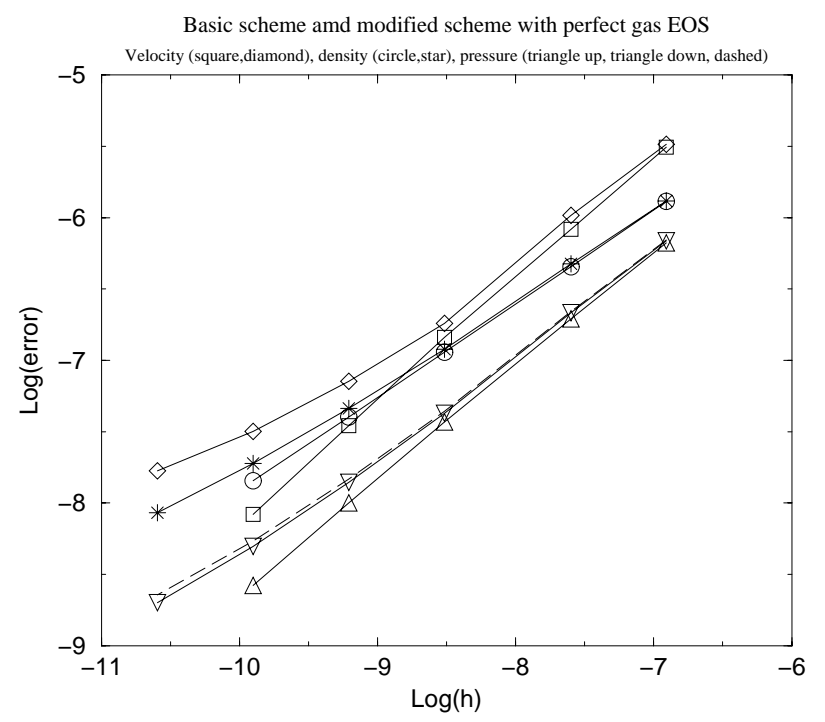

Figure 14. Perfect gas EOS: approximate decomposition.

\subsubsection{Approximate decomposition}

We set here $\epsilon=0.1$ and:

$$
\left\{\begin{array}{l}
\phi_{1}(\rho, P, C, \psi)=(1-\epsilon) \frac{P}{\gamma_{1}-1}, \\
\phi_{3}(\rho, P, C, \psi)=\epsilon \frac{P}{\gamma_{1}-1} .
\end{array}\right.
$$

When focusing on the standard Sod shock tube problem which involves one 3 -shock wave, and using meshes with up to 40000 nodes, the error in $L^{1}$ norm has been plotted in Figure 14. While linear rate of convergence is achieved when using the correct decomposition (velocity (squares), pressure (triangles up), density (circles)), and thus the fully unmodified conservative scheme (see also [18]), the measured error associated with the hybrid scheme (velocity (diamonds), pressure (triangles down), density (stars)) diminishes much slower on finer meshes. Actually, detailed qualitative investigations around the numerical shock locations show that both are separated by an $O(1)$ length, which can hardly be seen unless the mesh contains more than 10000 nodes, which is seldomly examined in pratice of course. This result confirms investigation of EOS in $T_{3}$ (Van der Waals) described previously. This is also confirmed in a "continuous" way by the next numerical experiment.

\subsubsection{Wrong decomposition}

We set here $\epsilon=1$, thus:

$$
\phi_{1}=0 \quad \text { and } \quad \phi_{3}=\frac{P}{\gamma_{1}-1}
$$

Updating the cell pressure at the end of the time step is performed through:

$$
P_{i}^{n+1}=\left(\gamma_{1}-1\right)\left(\phi_{3}\right)_{i}^{n+1}
$$

We provide below some comparison of both approximations, using a coarse mesh with two hundred nodes and a fine mesh with 10000 nodes. It obviously appears that the hybrid scheme no longer converges towards the correct solution (Fig. 15). Actually zooming the approximate solution provided by schemes with 5000 and 

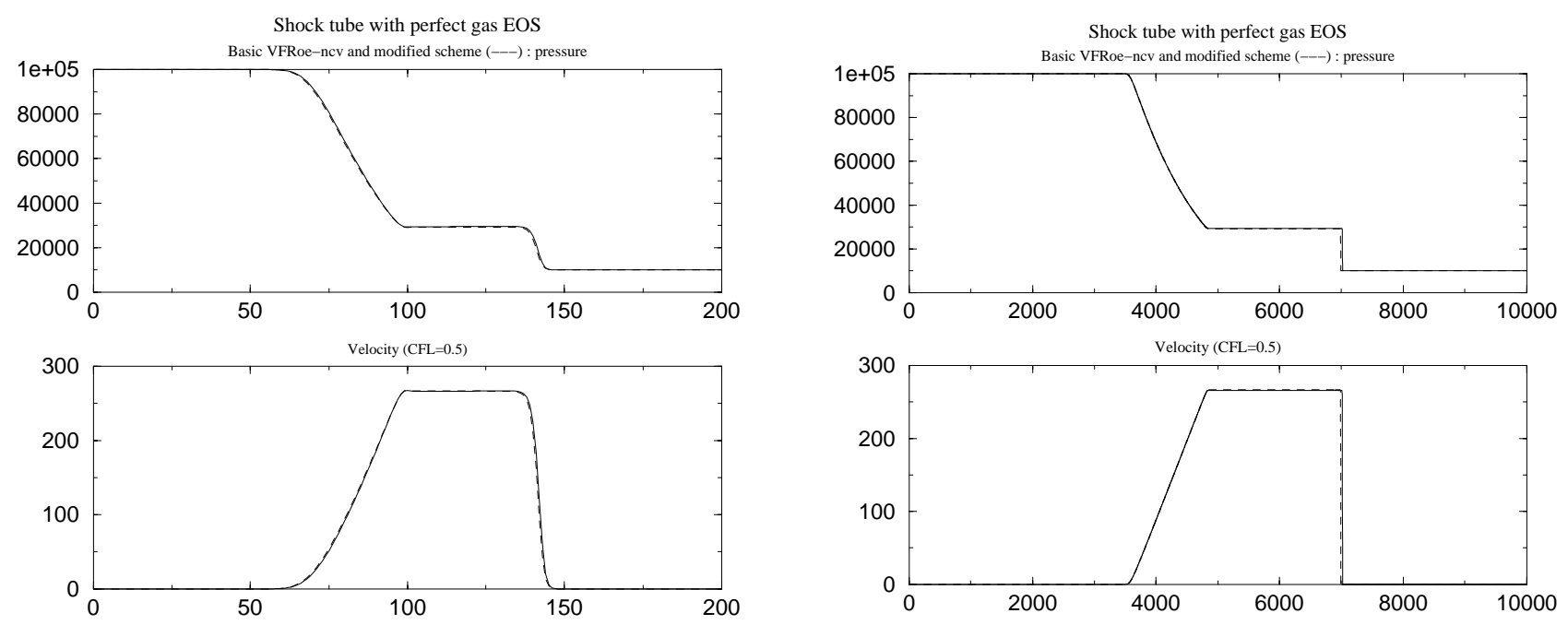

Figure 15. Perfect gas EOS: correct and wrong decomposition - coarse (left) and fine mesh (right).
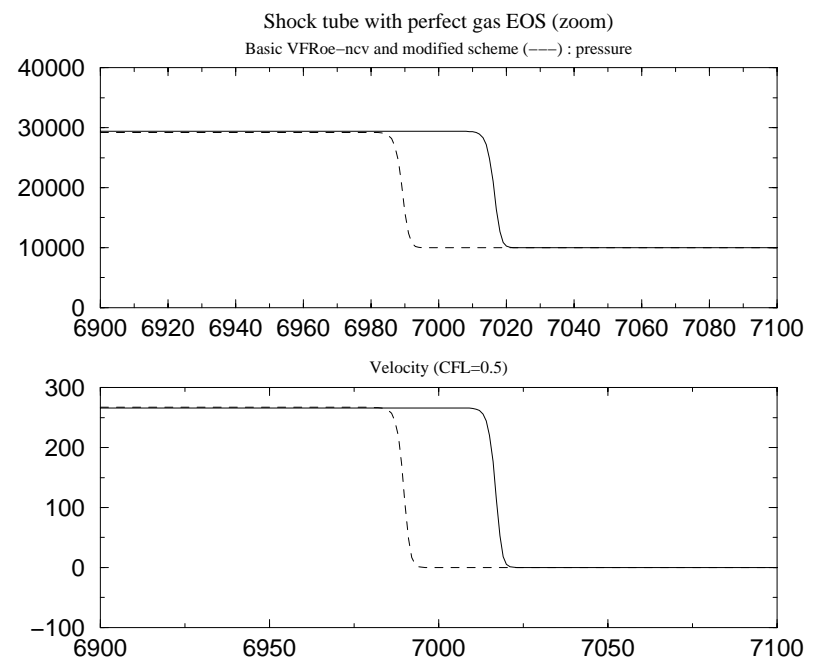

FiguRE 16. Perfect gas EOS: correct and wrong decomposition - finer mesh (zoom).

10000 cells enables to check that the number of nodes between the two locations of 3 shock waves doubles when refining the mesh by two (Fig. 16). This is confirmed by computations on finer meshes. Of course the error still seems to be negligible on coarse meshes. Results are here in agreement with [24].

\section{A BLEND SCHEME}

We propose the following overall strategy, which relies on tuning of both the original conservative scheme to deal with fine meshes, and the above mentioned scheme to benefit from pure representation of moving contact discontinuities on coarse meshes. It simply requires some parametric function in order to switch from one scheme to the other when the mesh is refined, and of course when complex EOS are considered. Thus, the cell 


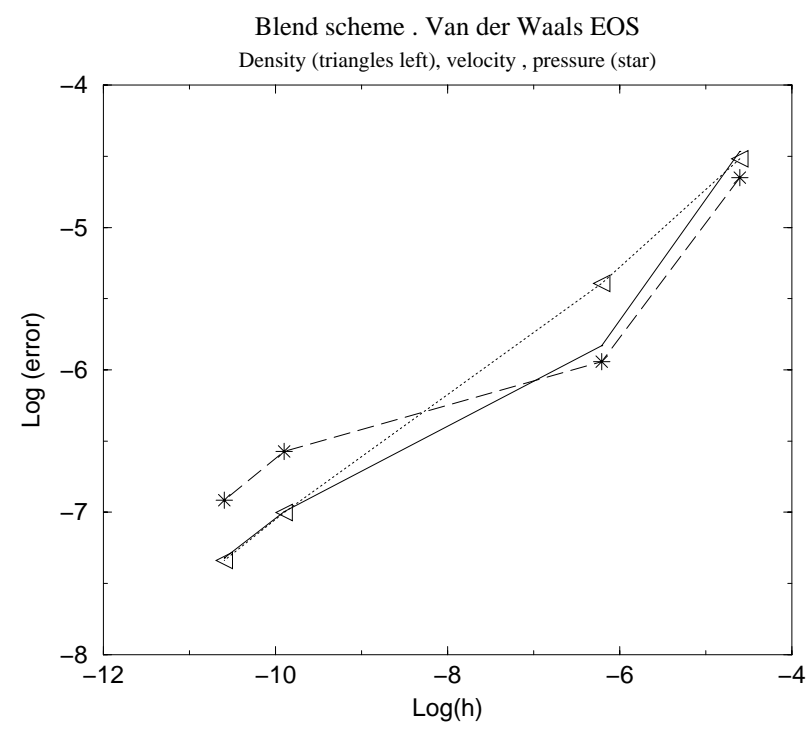

Figure 17. Van der Waals EOS: error in $L^{1}$ norm - 3-shock wave.

pressure which will be used in practice will be $p_{i}^{n+1}$ :

$$
\begin{aligned}
& P_{i}^{n+1}=P\left(\rho_{i}^{n+1}, e_{i}^{n+1}, C_{i}^{n+1}, \psi_{i}^{n+1}\right) \\
& p_{i}^{n+1}=\alpha(h) P_{i}^{n+1}+(1-\alpha(h)) \tilde{P}_{i}^{n+1} .
\end{aligned}
$$

where $\tilde{P}_{i}^{n}$ is given in (28), and $h$ stands for the mean mesh size. For given EOS in $T_{1} P_{i}^{n}=\tilde{P}_{i}^{n}$, thus the choice $\alpha(h)$ has no influence. Moreover, $\alpha(h)=0$ should be used for EOS in $T_{2}$. Otherwise, if the EOS is not in $T_{1} \cup T_{2}, \alpha(h)$ should comply with:

$$
\left\{\begin{array}{l}
\alpha(h)=1 \text { if } h \leq h_{0}, \\
\alpha(h)=0 \text { if } h \geq h_{1},
\end{array}\right.
$$

for given mesh sizes $h_{0}<h_{1}$ provided by user.

In practice, standard conservative schemes correspond to the formal choice $h_{0}=h_{1}=+\infty$, whereas the so-called hybrid scheme corresponds to $h_{0}=h_{1}=0$. Numerical tests reported above suggest some practical values. A first natural idea is to minimize the error in $L^{1}$ norm on any mesh, which is of interest for industrial purposes. Some way to achieve that is to define $h_{0}$ as the mesh size for which the error of the conservative scheme is equal to the error of its hybrid modification, and $h_{1}$ as the mesh size beyond which the error for the non conservative scheme remains constant. This of course would require computing both schemes and even more the error, which obviously is out of reach in practice. A practical example is given below (see Fig. 17), which consists in computing approximations of Euler equations with Van der Waals EOS and initial condition such that a strong 3 -shock wave develops. We have chosen here a linear path to connect $h_{0}=10^{-4}$ and $h_{1}=1 / 200$ through $\alpha(h)=\frac{h_{1}-h}{h_{1}-h_{0}}$ in the interval $\left(h_{0}, h_{1}\right)$. The error in $L^{1}$ norm is decreasing, but certainly not optimal, since both $h_{0}$ and $h_{1}$ are upper bounds of optimal values which are approximately for that case $h_{0}^{\text {opt }}=10^{-5}$ and $h_{1}^{\text {opt }}=10^{-4}$. Another point is that the choice of the path $\alpha(h)$ only influences the accuracy in the inner range $\left(h_{0}, h_{1}\right)$, which does not seem to be important in practice, at least in a multidimensional framework, due to the fact that $3 \mathrm{D}$ industrial meshes contain at most a few million cells, which corresponds to a characteristic mesh size $h=1 / 100$ in a one dimensional frame, and thus is obviously greater than $h_{1}$. 


\section{CONCLUSiOn}

This paper is devoted to the computation of Euler equations in a one-dimensional framework, with arbitrary equation of state, assuming the internal energy depends on pressure and density variables, but also on concentrations of some species and a colour function. It has been shown that when focusing on exact or adequate approximate Godunov solvers, one needs to distinguish three different classes of EOS. One thus needs to compute some redundent information (from a continuous point) in order to cope with the second and third class. Actually, one needs first to decompose the internal energy into three terms which respectively belong to the three classes. Afterwards, one needs to compute an extra (respectively two) equation(s) when some contribution occurs in the second or third class (respectively in both second and third class) in the decomposition.

Some schemes have been proposed to compute the latter non conservative governing equations in addition to the first five conservative equations associated with total mass, mass of species, total momentum, total energy and colour function. Thus pure unsteady contact discontinuities are very well predicted on coarse meshes when using the so-called hybrid scheme. We now may sum up the main results.

On the basis of a series of numerical tests including shocks:

1. using discrete $L^{1}$ measure of error, we have provided numerical evidence that the basic Godunov scheme and a broad class of approximate Godunov schemes (VFRoe-ncv schemes using $Y$ in (6)) converge towards the right solution for any of EOS in $T_{1} \cup T_{2} \cup T_{3}$ which have been tested (the rate of convergence exhibits that both $U, P$ converge as $h$ for EOS in $T_{1}$-otherwise $h^{\frac{1}{2}}$ - towards the right solution, while concentration or density converge as $h^{\frac{1}{2}}$ for any EOS);

2. the new proposal of modified exact or approximate "Godunov" schemes (27) identifies with a classical conservative formulation when the EOS is in $T_{1}$, and converges towards the right solution when the EOS lies in $T_{2}$, with indeed much increased accuracy on coarse meshes when compared to classical conservative schemes, (rate of convergence for modified "Godunov" schemes exhibits that both $U, P$ converge as $h$ towards the right solution - concentration or density still converge as $h^{\frac{1}{2}}-$ );

3. the modified exact or approximate "Godunov" schemes (27) enable to reach increased accuracy on coarse meshes with respect to standard conservative schemes when EOS have a non zero contribution in $T_{3}$, though they converge towards wrong shock solutions when using huge mesh refinement (approximately more than 20000 cells in a $1 D$ framework, which is beyond the commonly used range of meshes, see [11] and $[2])$.

This may on the whole motivate the use of the blend scheme (Sect. 8) which benefits from nice approximations on coarse meshes of the hybrid scheme, and still inherits the property of convergence towards the right solution on finer meshes. In practice, this will in fact correspond to the use of the hybrid scheme since very few meshes contain more than $\left(10^{2}\right)^{3}$ cells in an industrial computation and none contains more than $\left(2.10^{4}\right)^{3}$ cells nowadays. The hybrid scheme is thus appealing for industrial purposes since it not only enables to increase accuracy on given (coarse) mesh size, but also enables to reduce CPU time due to the fact that computation of pressure is

usually much faster when computing modified pressure $\tilde{P}$ rather than standard value $P\left(\rho_{i}^{n}, e_{i}^{n}, C_{i}^{n}, \psi_{i}^{n}\right)$. This is actually the case when applying Chemkin database, which only requires an algebraic calculus instead of a Newton procedure to compute cell pressure at the end of the time step, but also when dealing with more complex EOS or tabulated EOS as suggested. It is emphasised that this remark takes into account the fact that two additional discrete equations for redundent information must be computed.

It seems clear that this work is not only useful in the framework of two-phase flow modelling with help of single fluid models of the Euler type, but also when retaining the two-fluid two-pressure approach. In an ongoing work, possible extensions to multidimensional unstructured meshes are investigated.

\section{APPEndiX A. VFRoE-NCV SCHEMES FOR SySTEMS OF CONSERVATION LAWS}

This appendix presents the construction of VFRoe-ncv schemes, focusing on systems of conservation laws. We reduce to the one dimensional case, with regular meshes (the extension to the multidimensional case and to 
unstructured meshes is classical). Following notations introduced in the body of the present paper, we denote $W: \mathbb{R}_{+} \times \mathbb{R} \rightarrow \mathbb{R}^{n}$ the exact solution of the non degenerate hyperbolic system:

$$
\left\{\begin{array}{l}
\frac{\partial W}{\partial t}+\frac{\partial F(W)}{\partial x}=0 \\
W(0, x)=W_{0}(x) .
\end{array}\right.
$$

Let $\Phi$ be a regular invertible function from $\mathbb{R}^{n}$ to $\mathbb{R}^{n}$ and $\Psi$ its inverse. If $W$ is a regular solution of the above system, then $Y=\Phi(W)$ is solution of

$$
\frac{\partial Y}{\partial t}+B(Y) \frac{\partial Y}{\partial x}=0
$$

where $B(Y)=(D \Psi(Y))^{-1}(D F(\Psi(Y)))(D \Psi(Y))$.

As mentioned above, VFRoe-ncv schemes are approximate Godunov schemes. Hence, they may be written in the form

$$
h_{i}\left(W_{i}^{n+1}-W_{i}^{n}\right)+\delta t\left(F\left(\Psi\left(Y_{i+1 / 2}^{*}\right)\right)-F\left(\Psi\left(Y_{i-1 / 2}^{*}\right)\right)\right)=0
$$

We describe now the computation of $Y_{i+1 / 2}^{*}$. The state $Y_{i+1 / 2}^{*}$ corresponds to the exact solution $Y^{*}$ at $x=0$ of the linearized hyperbolic system:

$$
\left\{\begin{array}{l}
\frac{\partial Y^{*}}{\partial t}+B(\hat{Y}) \frac{\partial Y^{*}}{\partial x}=0 \\
Y^{*}(0, x)=\left\{\begin{array}{l}
Y_{L}=\Phi\left(W_{i}^{n}\right) \\
Y_{R}=\Phi\left(W_{i+1}^{n}\right) \text { if } x>0
\end{array}\right.
\end{array}\right.
$$

where $\hat{Y}=\left(Y_{L}+Y_{R}\right) / 2$. Since $Y^{*}(t, x)$ is the solution of a linear system, its computation is classical. Setting $\beta=\frac{x}{t}$, we have $Y^{*}(t, x)=\varphi\left(\beta, Y_{L}, Y_{R}\right)$ :

$$
\begin{aligned}
\varphi\left(\beta ; Y_{L}, Y_{R}\right) & =Y_{L}+\sum_{\beta>\widetilde{\lambda_{k}}}\left({ }^{t} \widetilde{l_{k}} \cdot\left(Y_{R}-Y_{L}\right)\right) \widetilde{r_{k}} \\
& =Y_{R}-\sum_{\beta<\widetilde{\lambda_{k}}}\left({ }^{t} \widetilde{l_{k}} \cdot\left(Y_{R}-Y_{L}\right)\right) \widetilde{r_{k}}
\end{aligned}
$$

where $\widetilde{l_{k}}, \widetilde{\lambda_{k}}$ and $\widetilde{r_{k}}, k=1, \ldots, n$, are respectively left eigenvectors, eigenvalues and right eigenvectors of matrix $B(\hat{Y})$. Thus, we have

$$
Y_{i+1 / 2}^{*}=\varphi\left(0 ; Y_{L}, Y_{R}\right)
$$

\section{REFERENCES}

[1] R. Abgrall, How to prevent pressure oscillations in multicomponent flow calculations: a quasi conservative approach. $J$. Comput. Phys. 125 (1995) 150-160.

[2] R. Abgrall and S. Karni, Computations of compressible multifluids. J. Comput. Phys. 169 (2001) 594-623.

[3] G. Allaire, S. Clerc and S. Kokh, A five equation model for the simulation of interfaces between compressible fluids. J. Comput. Phys. 181 (2002) 577-616. 
[4] G. Allaire, S. Clerc and S. Kokh, A five equation model for the numerical solution of interfaces in two phase flows. C.R. Acad. Sci. Paris Sér. I Math. 331 (2000) 1017-1022.

[5] T. Barberon, P. Helluy and S. Rouy, Finite Volume simulations of cavitating flows, in Proc. of Third Symposium on Finite Volumes for Complex Applications, R. Herbin and D. Kroner Eds., Hermes Penton Science (2002) 455-462.

[6] M. Barret, E. Faucher and J.M. Hérard, Some schemes to compute unsteady flashing flows. AIAA J. 40 (2002) 905-913.

[7] S. Bilicki and D. Kardas, Approximation of thermodynamic properties for subcooled water and superheated steam. Polish Academy of Sciences (1991).

[8] S. Bilicki and J. Kestin, Physical aspects of the relaxation model in two phase flows. Proc. Roy. Soc. London A 428 (1990) 379-397.

[9] S. Bilicki, J. Kestin and M.M. Pratt, A reinterpretation of the results of the moby dick experiments in terms of the non equilibrium model. J. Fluid Eng. 112 (1990) 212-217.

[10] T. Buffard, T. Gallouët and J.M. Hérard, Schéma VFRoe en variables caractéristiques. Principe de base et applications aux gaz réels. EDF-DER Report HE-41/96/041/A (1996) in French.

[11] T. Buffard, T. Gallouët and J.M. Hérard, A sequel to a rough Godunov scheme. Application to real gas flows. Comput. \& Fluids 29 (2000) 813-847.

[12] M. Buffat and A. Page, Extension of Roe's solver for multi species real gases. LMFA report, École Centrale de Lyon, Lyon, France (1995).

[13] S. Clerc, Accurate computation of contact discontinuities in flows with general equations of state. Comput. Methods Appl. Mech. Engrg. 178 (1999) 245-255.

[14] S. Clerc, Numerical simulation of the homogeneous equilibrium model for two phase flows. J. Comput. Phys. 161 (2000) $354-375$.

[15] F. Coquel and B. Perthame, Relaxation of energy and approximate Riemann solvers for general pressure laws in fluid dynamics equations. SIAM J. Numer. Anal. 35 (1998) 2223-2249 (in Memory of Ami Harten).

[16] E. Faucher, J.M. Hérard, M. Barret and C. Toulemonde, Computation of flashing flows in variable cross-section ducts. Int. J. Comput. Fluid Dyn. 13 (2000) 365-391.

[17] R.P. Fedkiw, T. Aslam, B. Merriman and S. Osher, A non oscillatory eulerian approach to interfaces in multimaterial flows (the ghost fluid approach). J. Comput. Phys. 152 (1999) 457.

[18] T. Gallouët, J.M. Hérard and N. Seguin, Some recent Finite Volume schemes to compute Euler equations using real gas EOS. Internat. J. Numer. Methods Fluids 39-12 (2002) 1073-1138.

[19] T. Gallouët, J.M. Hérard and N. Seguin, An hybrid scheme to compute contact discontinuities in Euler systems. LATP Report 01-027, Université de Provence, France (2001).

[20] T. Gallouët, J.M. Hérard and N. Seguin, On the use of some symmetrizing variables to deal with vacuum (submitted).

[21] S. Gavrilyuk and R. Saurel, Mathematical and numerical modelling of two phase compressible flows with inertia. J. Comput. Phys. 175 (2002) 326-360.

[22] E. Godlewski and P.A. Raviart, Numerical approximation for hyperbolic systems of conservation laws. Springer Verlag (1996).

[23] S.K. Godunov, A difference method for numerical calculation of discontinous equations of hydrodynamics. Sbornik (1959) 271-300 (in Russian).

[24] X. Hou and P. G. Le FLoch, Why non conservative schemes converge to wrong solutions. Math. Comp. 62 (1994) $497-530$.

[25] A. In, Numerical evaluation of an energy relaxation method for inviscid real fluids. SIAM J. Sci. Comput. 21 (1999) 340-365.

[26] A. In, Méthodes numériques pour les équations de la dynamique des gaz complexes et écoulements diphasiques. Ph.D. thesis, Université Paris VI, France (1999).

[27] M. Ishii, Thermo-fluid dynamic theory of two-phase flows. Collection de la Direction des Etudes et Recherches d'Electicité de France (1975).

[28] A.K. Kapila, S.F. Son, J.B. Bdzil, R. Menikoff and D.S. Stewart, Two-phase modelling of DDT: structure of the velocity relaxation zone. Phys. Fluids 9 (1997) 3885-3897.

[29] S. Karni, Multicomponent flow calculations by a consistent primitive algorithm. J. Comput. Phys. 112 (1994) $31-43$.

[30] S. Karni, Hybrid multifluid algorithms. SIAM J. Sci. Comput. 17 (1996) 1019-1039.

[31] S. Karni and R. Abgrall, Ghost fluid for the poor: a single fluid algorithm for multifluid. Oberwolfach (2001).

[32] R. Kee, J. Miller and T. Jefferson, Chemkin: a general purpose, problem independant transportable fortran chemical kinetics code package. SAND Report 80-8003, Sandia National Laboratories.

[33] S. Kokh, Aspects numériques et théoriques de la modélisation des écoulements diphasiques compressibles par des méthodes de capture d'interface. Ph.D. thesis, Université Paris VI, France (2001).

[34] F. Lagoutière, Modélisation mathématique et résolution numérique de problèmes de fluides compressibles à plusieurs constituants. Ph.D. thesis, Université Paris VI, France (2000).

[35] A. Letellier and A. Forestier, Le problème de Riemann en fluide quelconque. CEA-DMT Report 93/451 (1993) in French.

[36] R. LeVeque, Numerical methods for conservation laws. Birkhauser (1992).

[37] R. Pollak, Die thermodynamischen eigenschaften von wasser dargestellt durch eine kanonische zustands gleichung fur die fluiden homogenen und heterogenen zustande bis 1200 Kelvin und 3000 bars. Ph.D. thesis, Ruhr Universitat, Germany (1974). 
[38] P. Rascle and O. Morvant, Interface utilisateur de Thetis - THErmodynamique en Tables d'InterpolationS. EDF-DER Report HT-13/95021B, Clamart, France (1995) in French.

[39] P.L. Roe, Approximate Riemann solvers, parameter vectors and difference schemes. J. Comput. Phys. 43 (1981) $357-372$.

[40] S. Rouy, Modélisation mathématique et numérique d'écoulements diphasiques compressibles. Ph.D. thesis, Université de Toulon et du Var, France (2000)

[41] R. Saurel and R. Abgrall, A multiphase Godunov method for compressible multifluid and multiphase flows. J. Comput. Phys. 150 (1999) 425-467.

[42] R. Saurel and R. Abgrall, A simple method for compressible multifluid flows. SIAM J. Sci. Comput. 21 (1999) 1115-1145.

[43] J. Sethian, Level set methods. Cambridge University Press (1996).

[44] K.M. Shyue, A fluid mixture type algorithm for compressible multicomponent flow with Van der Waals equation of state. $J$. Comput. Phys. 156 (1999) 43-88.

[45] J. Smoller, Shock waves and reaction diffusion equations. Springer Verlag (1983).

[46] E.F. Toro, Riemann solvers and numerical methods for fluid dynamics. Springer Verlag (1997).

[47] I. Toumi, Contribution à la modélisation numérique des écoulements diphasiques eau-vapeur. Thèse d'habilitation, Université Paris Sud, France (2000). 